Aus der Klinik für Augenheilkunde

(Prof. Dr. med. H. Hoerauf)

der Medizinischen Fakultät der Universität Göttingen

\title{
Prospektive Evaluation kardiovaskulärer Risikofaktoren bei Patienten mit venösen Gefäßverschlüssen im Auge
}

\author{
INAUGURAL-DISSERTATION \\ zur Erlangung des Doktorgrades \\ der Medizinischen Fakultät der \\ Georg-August-Universität zu Göttingen
}

vorgelegt von

Janina Monika Best

aus

Bonn

Göttingen 2015 
Dekan:

I. Berichterstatter: Prof. Dr. Nicolas Feltgen

II. Berichterstatter/in: PD Dr. Miriam Puls

III. Berichterstatter/in: Prof. Dr. George Trendelenburg

Tag der mündlichen Prüfung: $\quad$ 16.02.2016 


\section{Inhaltsverzeichnis}

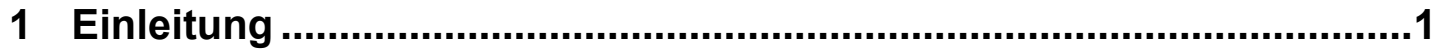

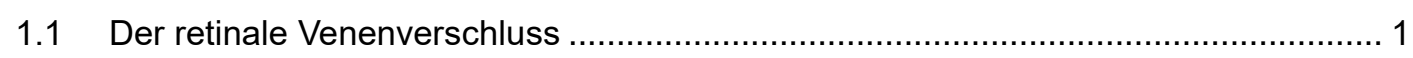

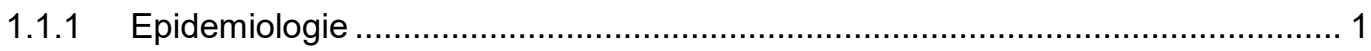

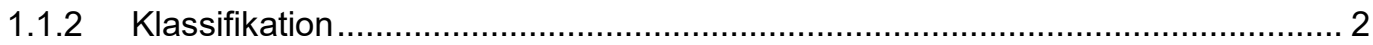

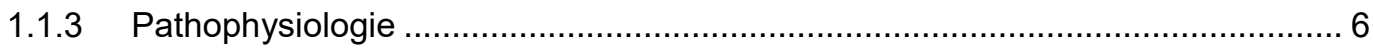

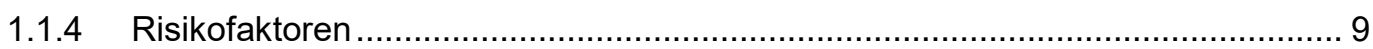

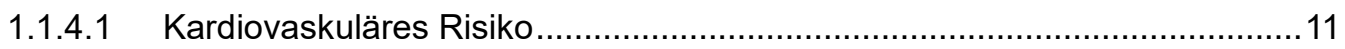

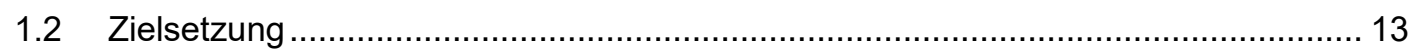

2 Patienten und Methoden .......................................................................14

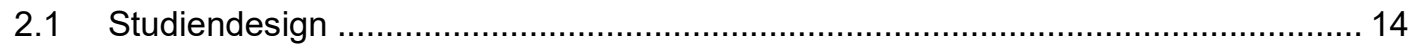

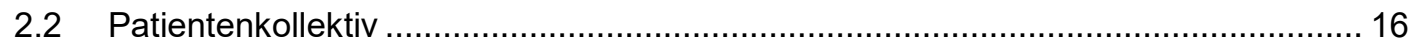

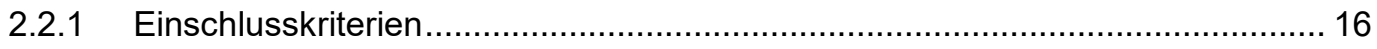

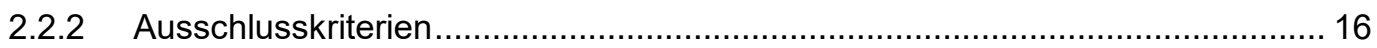

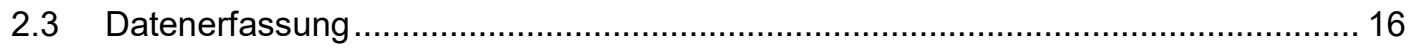

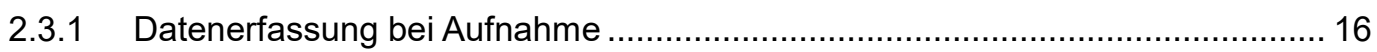

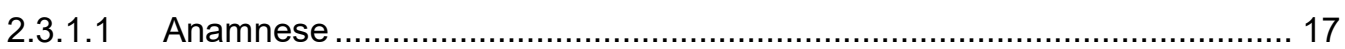

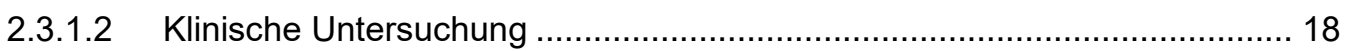

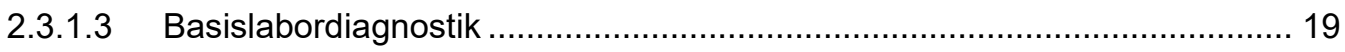

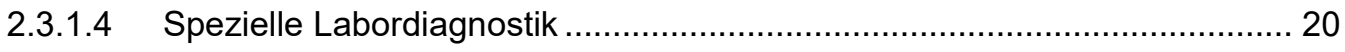

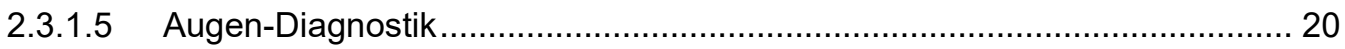

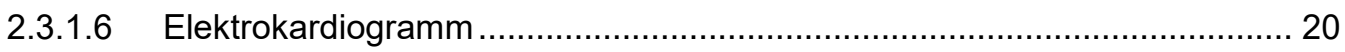

2.3.2 Datenerhebung während des stationären Aufenthaltes .................................. 21

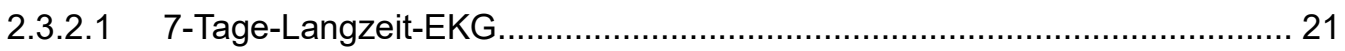

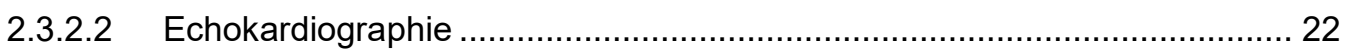

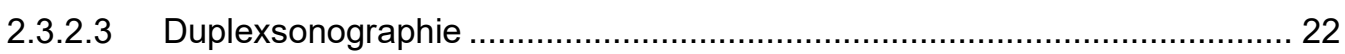

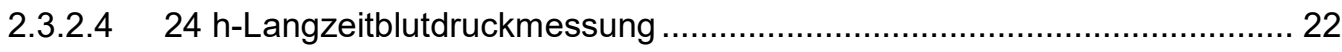

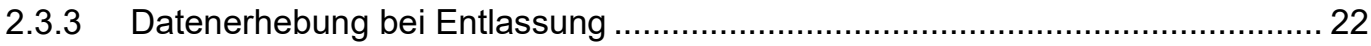




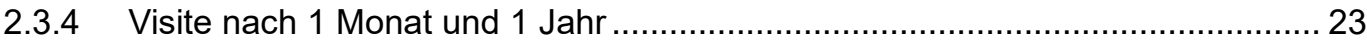

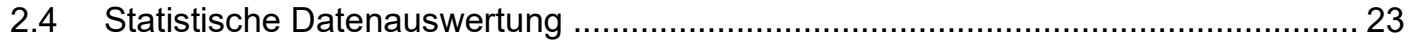

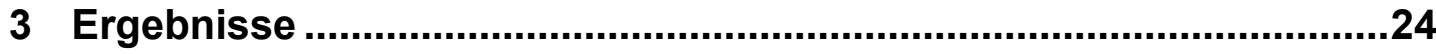

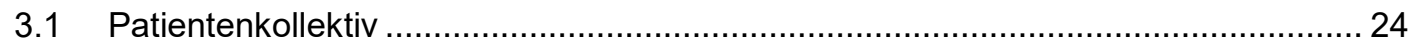

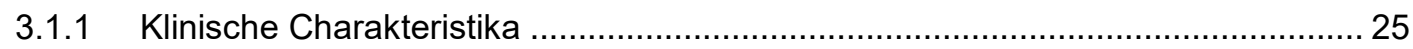

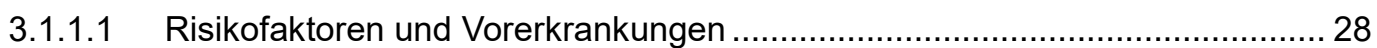

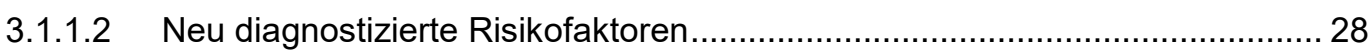

3.1.1.3 Bekannte und neu diagnostizierte Risikofaktoren ............................... 30

3.2 Vergleich einzelner Prävalenzen von Risikofaktoren bei Patienten mit anderen

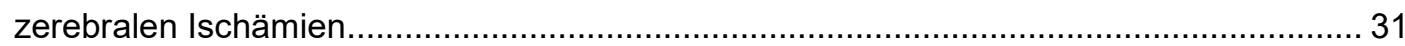

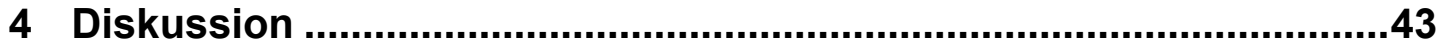

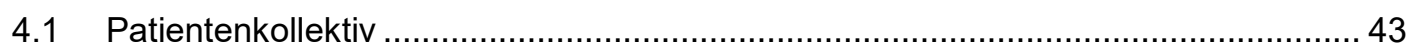

4.2 Evaluation kardiovaskulärer Risikofaktoren ................................................. 44

4.3 Vergleich einzelner Prävalenzen von Risikofaktoren bei Patienten mit anderen

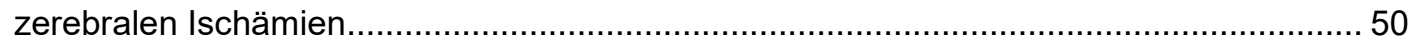

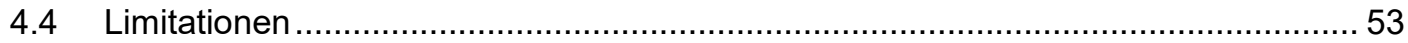

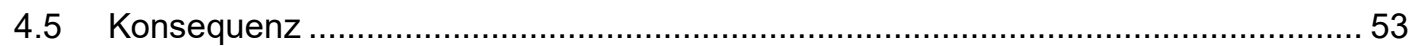

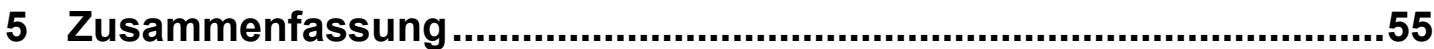

6 Anhang ......................................................................................57

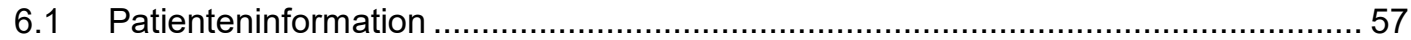

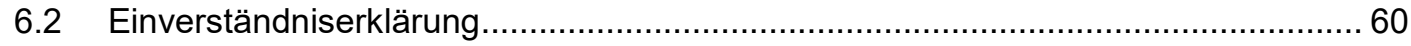

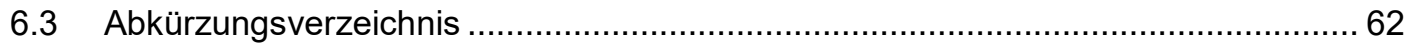

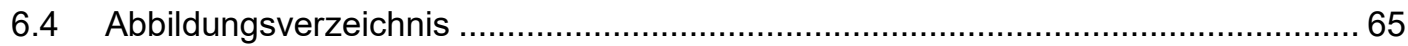

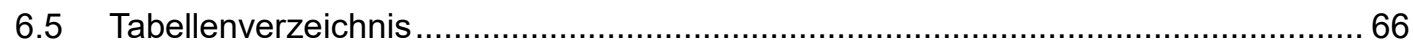

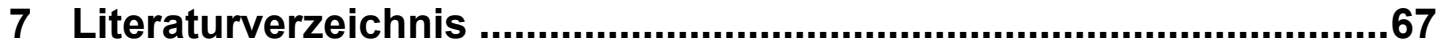

8 Präsentationen ......................................................................................75 


\section{Einleitung}

\subsection{Der retinale Venenverschluss}

\subsubsection{Epidemiologie}

Retinale Venenverschlüsse sind die häufigste primäre Mikrozirkulationsstörung des Auges und nach der diabetischen Retinopathie die zweithäufigste vaskuläre Netzhauterkrankung (Lang 2007; Kuhli-Hattenbach et al. 2011; Seidel et al. 2014).

Der Erkrankungsgipfel liegt zwischen 60 und 70 Jahren (Dithmar et al. 2003), auch wenn die Wahrscheinlichkeit, einen retinalen Venenverschluss zu erleiden, mit zunehmendem Alter steigt (Rogers et al. 2010a). Ein Venenastverschluss ist ca. 3-mal häufiger als ein Zentralvenenverschluss (David et al. 1988). Der retinale Venenverschluss betrifft beide Augen gleichermaßen (Klein 2008). Männer sind häufiger betroffen als Frauen (Hayreh 1994; Dithmar et al. 2003). Die Prävalenz eines retinalen Venenverschlusses liegt bei 0,7 \% (0,6 \% VAV, 0,1\% ZVV) (Klein et al. 2000) bis $1,1 \%(0,9 \% \mathrm{VAV}, 0,2 \% \mathrm{ZVV})$ und ist in verschiedenen ethnischen Gruppen ähnlich (Cheung et al. 2008). Bei Lateinamerikanern und Asiaten ist sie etwas höher als in der weißen und schwarzen Bevölkerung (Rogers et al. 2010a; Cheung et al. 2008). Die fünf-Jahres Inzidenz beträgt 0,8 \% (0,6 \% VAV, 0,2 \% ZVV) (Klein et al. 2000). Nach zehn Jahren liegt die Inzidenz bei 1,6 \% (1,2 \% VAV, 0,4 \% ZVV inklusive HZVV) (Cugati 2006) und nach 15 Jahren bei 2,3 \% (1,8 \% VAV, 0,5\% ZVV) (Klein 2008). Das Risiko, einen erneuten Verschluss am gleichen Auge zu bekommen, liegt bei 0,9 \% in zwei Jahren und bei 2,5 \% in vier Jahren, am anderen Auge jedoch deutlich höher bei $7,7 \%$ in zwei Jahren und bei 11,9 \% in vier Jahren (Hayreh 1994). 


\subsubsection{Klassifikation}

Die retinalen Gefäße verlaufen in sehr enger Nachbarschaft zueinander und treten am Augenfundus als Zentralarterie oder Zentralvene aus den Sehnerven hervor. Dort teilen sie sich in zwei Hauptstämme nach oben und unten mit jeweils zwei großen Ästen nach temporal und nasal auf (Marcucci et al. 2011).

Venenverschlüsse werden klinisch sowohl nach Ausdehnung als auch nach Ischämiegrad eingeteilt (Dithmar et al. 2003; Hayreh 2005).

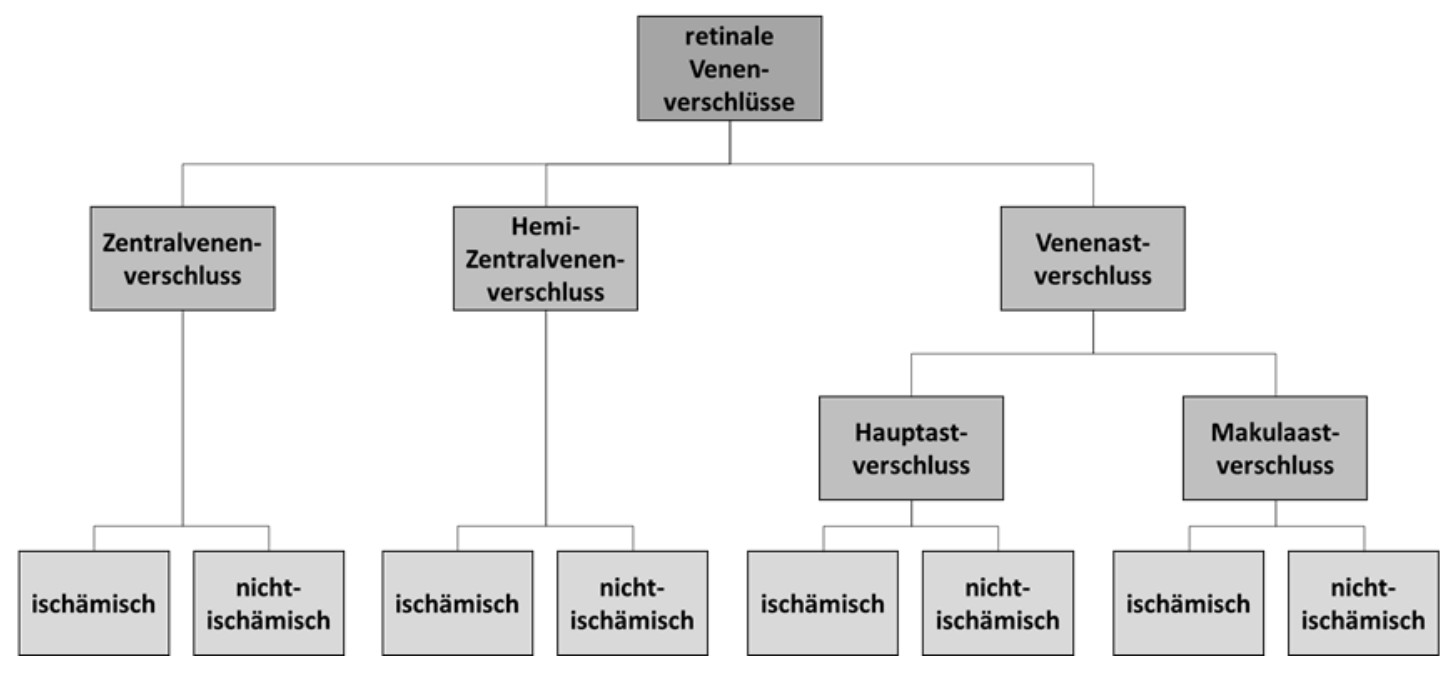

Abbildung 1: Einteilung retinale Venenverschlüsse 
Der Zentralvenenverschluss ist charakterisiert durch streifen- oder punktförmige Blutungen in allen vier Netzhautquadranten (Dithmar et al. 2003). Für die Einteilung des Ischämiegrades benötigt man eine Fluoreszeinangiographie. Erfolgt die Einteilung nach Ischämiegrad, unterscheidet man den nicht-ischämischen Verschluss vom ischämischen Verschluss, die Übergangsform wird als, intermediärer Typ ' bezeichnet. Wenn die Blutperfusion in einer bestimmten Fläche gestört ist, spricht man von einem ischämischen Verschluss. Die Fläche wird anhand der Fläche des Sehnervenkopfes (Papille) abgeschätzt und liegt beim Venenastverschluss bei 5 Papillendurchmessern (PD) und beim Zentralvenenverschluss bei 10 PD (Dithmar et al. 2003). Ein nicht-ischämischer Verschluss kann im Verlauf der Erkrankung in einen ischämischen Verschluss übergehen, dieser Anteil liegt bei ca. 30 \% innerhalb von 3 Jahren (Rogers et al. 2010b; Lang 2007). 

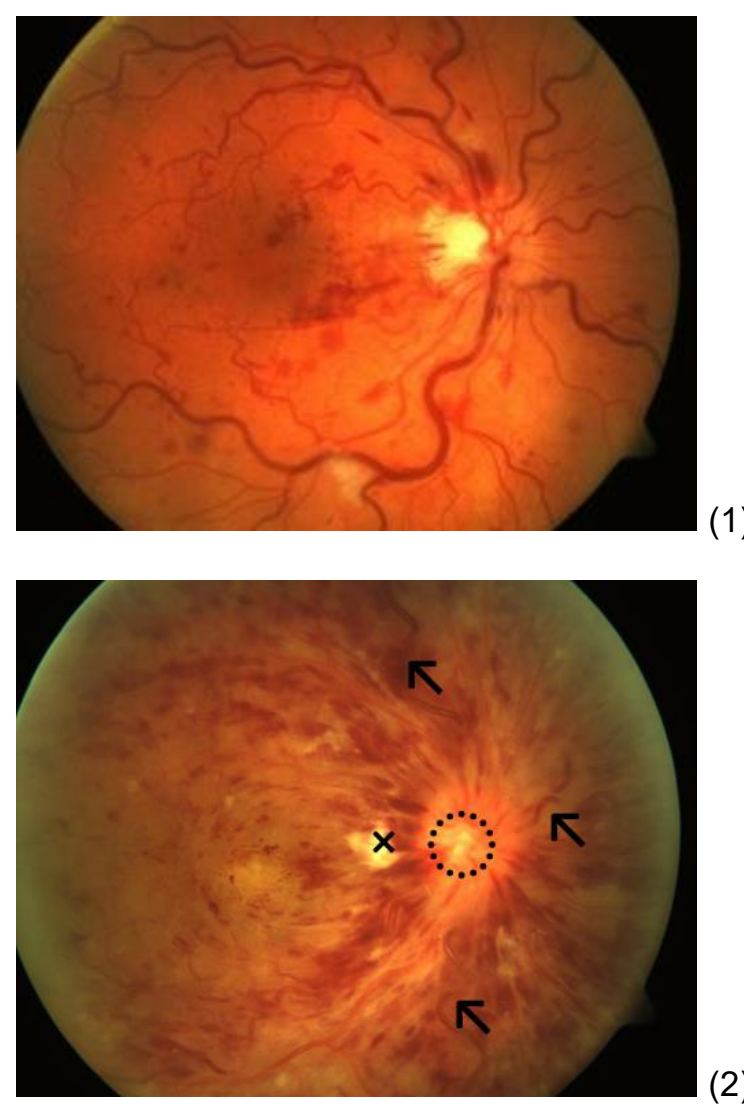

(1)

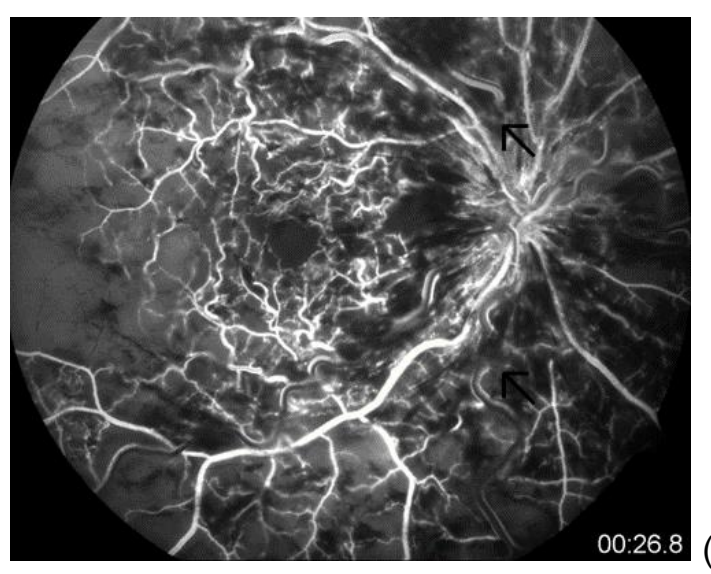

(2)

Abbildung 2: Zentralvenenverschlüsse

(1) nicht-ischämischer ZVV mit deutlicher venöser Stauung, geschlängelten Gefäßen und wenig Blutungen, (2) ischämischer ZVV mit flächigen und dunklen intraretinalen Hämorrhagien, Cotton-Wool-Herden ( $\times$ ), Papillenschwellung () und Tortuositas $(\nwarrow)$ und in der (3) Fluoreszeinangiographie sichtbarer unzureichender Anflutung der gestauten, geschlängelten Gefäße $(\nwarrow)$ 
Eine Variante des ZVV ist der Hemi-Zentralvenenverschluss (HZVV). Hierbei teilt sich die Zentralvene noch innerhalb des Sehnervens in zwei Äste auf (Hayreh 1980). Bei einem HZVV ist nur die Hälfte des Fundus betroffen (Marcucci et al. 2011).

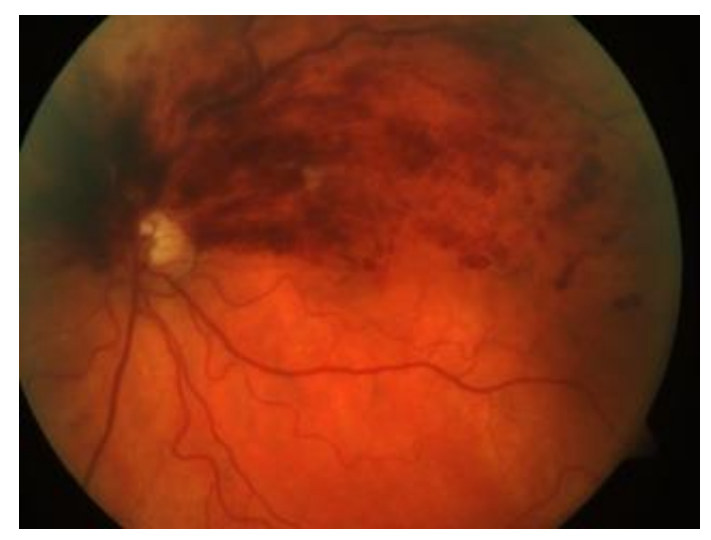

Abbildung 3: HZVV in der oberen Hälfte

mit flächigen retinalen Hämorrhagien

Der Venenastverschluss (VAV) wird in Hauptast- und Makulaastverschluss unterteilt (Dithmar et al. 2003). Beim Venenastverschluss ist in ca. $50 \%$ am häufigsten der obere äußere Quadrant mit der V. temporalis superior betroffen (Lang und Freissler 1992).

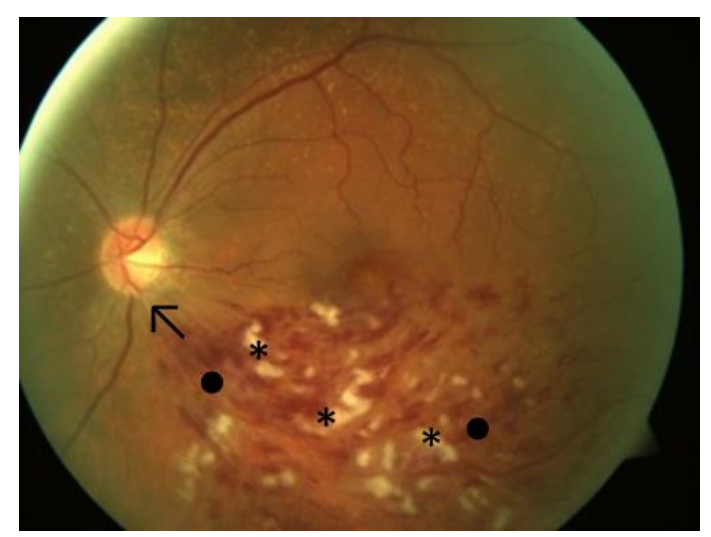

Abbildung 4: VAV der V. temporalis inferior

$\left(\nwarrow\right.$ Verschlussstelle) mit retinalen Hämorrhagien $(\bullet)$ und Cotton-Wool-Herden $\left({ }^{\star}\right)$ 


\subsubsection{Pathophysiologie}

Die genaue Entstehung eines retinalen Venenverschlusses ist nach wie vor unklar. In der Literatur werden verschiedene Ursachen diskutiert. Wichtig ist die eindeutige „pathophysiologische Abgrenzung des retinalen Venenverschlusses zu anderen thrombotischen Erkrankungen wie z.B. der tiefen Beinvenenthrombose“ (aus Feltgen et al. 2010, S. 682). Grundsätzlich gelten zwar die Regeln der Virchow-Trias (Veränderungen von Gefäßwand, Blutzusammensetzung und Strömungsgeschwindigkeit), der Thrombus kann, muss aber nicht, die primäre Ursache eines Verschlusses sein (Feltgen 2008). Patienten mit einem retinalen Venenverschluss weisen zudem die typischen vaskulären Risikofaktoren arterieller Gefäßkrankheiten auf (Feltgen et al. 2010). Dass diese Risikofaktoren zur Entstehung venöser retinaler Verschlüsse führen, lässt sich durch die enge Nachbarschaft zwischen retinalen Arterien und Venen gut erklären. Schon 1962 beschrieb Seitz, dass Gefäßwandveränderungen der Netzhautarterien zu Beeinträchtigungen des Blutstromes in den benachbarten Netzhautvenen führen (Seitz 1962).

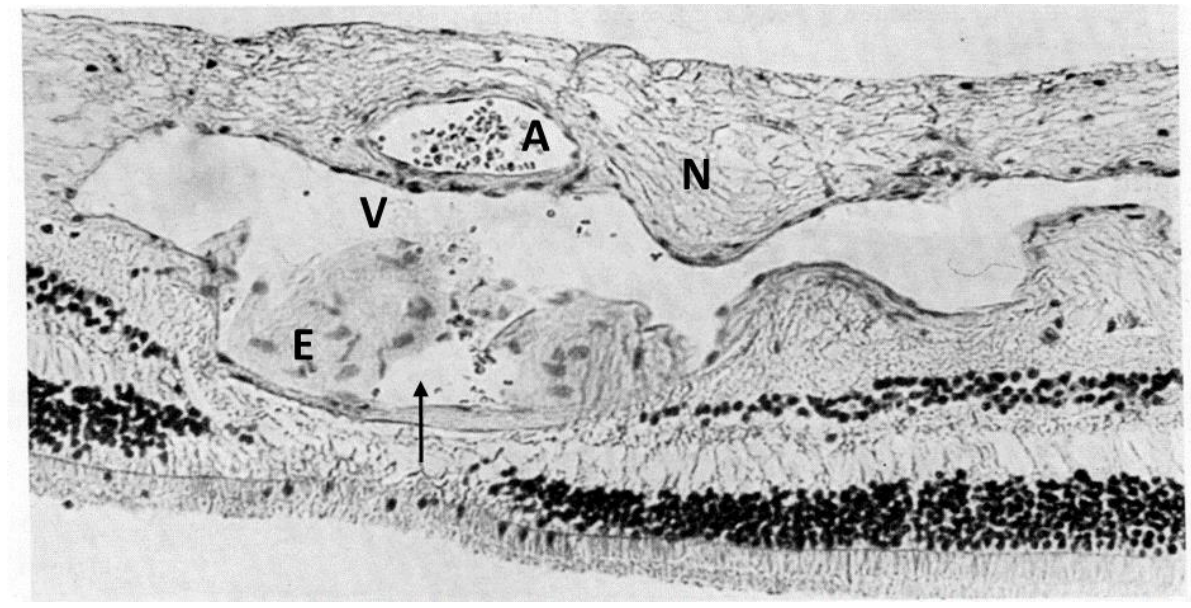

Abbildung 5: Histologischer Befund eines VAV

Das Präparat zeigt die Nachbarschaft von Arterie (A) und Vene (V) mit Gefäßwandaussackung (Pfeil), Endothelproliferation (E) und Netzhautödem (N) (aus Seitz 1962, S.74). 
Durch die daraus entstandene Verlangsamung der Strömungsgeschwindigkeit und Erhöhung der Blutviskosität resultiert sekundär ein partieller Thrombus der Netzhautvene (Seitz 1962). In der verstopften Vene kommt es durch den ungehinderten arteriellen Blutzustrom zur Erhöhung des intravasalen Druckes. Klinisch präsentiert sich eine gestaute, geschlängelte Vene (Tortuositas) (Feltgen et al. 2010). Die Folge ist eine Schrankenstörung mit retinalen Blutungen und Netzhautödem. Die behinderte Blutzirkulation führt zur Gewebehypoxie, die wiederum die Expression von permeabilitätssteigernden Botenstoffen, z.B. dem vascular endothelial growth factor (VEGF), fördert, welches die Ödembildung verstärkt. Die ischämiebedingte Expression der Botenstoffe führt zur pathologischen Gefäßneubildung (Neovaskularisation) in der Netzhaut (Feltgen 2008).

Eine weitere Ursache für die Entstehung retinaler Venenverschlüsse können die anatomischen Verhältnisse innerhalb des Sehnerven sein (Feltgen 2008). Umgeben von einer Lamina cribrosa verlaufen Zentralvene und Zentralarterie gemeinsam mit dem Nervus opticus im Zentralkanal. Eine Sklerosierung der Lamina cribrosa kann zur Kompression der Zentralvene führen (Dithmar et al. 2003). Ein Venenastverschluss tritt hingegen immer an arteriovenösen Kreuzungsstellen auf (Dithmar et al. 2003). Durch den Verlauf der pathologisch veränderten Netzhautarterie kommt es dann in diesen Abschnitten bevorzugt zu trophischen Störungen der Netzhautvene mit Verengung des Lumens und hämodynamischen Veränderungen im Gefäß (Seitz 1962). Dabei verläuft die Arterie meistens vor der Vene (Feist et al. 1992; Jefferies et al. 1993; Weinberg et al. 1990). 


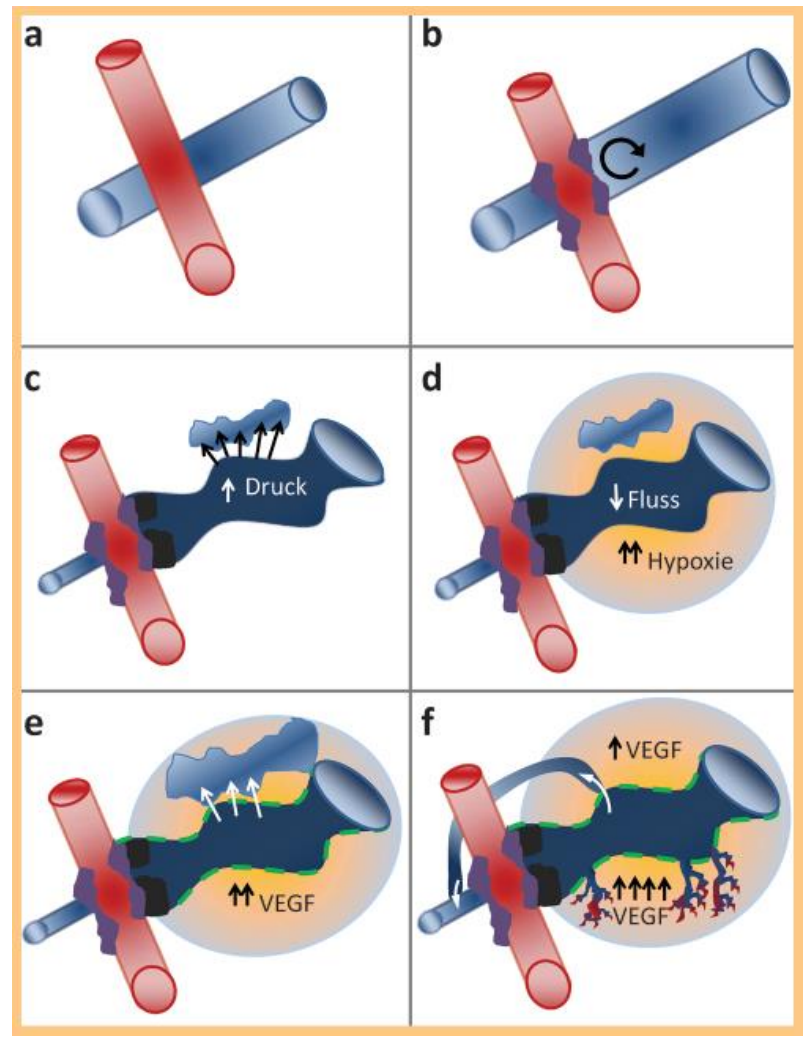

\section{Abbildung 6: Pathogenese des Venenastverschlusses mit typischen sekundären Komplikationen}

a) Ein Venenastverschluss entsteht typischerweise an Kreuzungsstellen von retinalen Arterien (rot) und Venen (blau). b) Wichtige Risikofaktoren sind arterielle Wandveränderungen, welche sekundär das Lumen der benachbarten Vene einengen und dort zu Strömungsunregelmäßigkeiten führen. c) Begünstigt durch den verlangsamten venösen Blutfluss kommt es zur Ausbildung eines partiellen Thrombus in der gestauten Vene. Dies führt zu einer weiteren Verlangsamung des venösen Abflusses, venösem Druckanstieg und Abpressen von Blut und Plasmabestandteilen in das umgebende Gewebe (Ödembildung). d) Gleichzeitig führt der verlangsamte venöse Blutfluss zu einer retinalen Zirkulationsstörung mit Ausbildung von Gewebshypoxie in der umgebenden Retina. e) Retinale Hypoxie wiederum führt zur Heraufregulation von VEGF, welches durch seine permeabilitätssteigernden Eigenschaften die Ödembildung verstärkt. f) Neben der Aufhebung der Blut-Retina-Schranke fördert VEGF auch die Ausbildung neuer Blutgefäße. In gewissen Grenzen kann dies für die Ausbildung von Kollateralkreisläufen förderlich sein (oben). Eine exzessive Heraufregulation von VEGF führt jedoch zur unkontrollierten Proliferation von Endothelzellen und zur Ausbildung pathologischer Proliferationsareale (unten) (aus Feltgen et al. 2010, S. 683 mit freundlicher Genehmigung). 


\subsubsection{Risikofaktoren}

Bei der Entstehung und dem Verlauf eines retinalen Venenverschlusses spielen kardiovaskuläre Risikofaktoren eine entscheidende Rolle. Im Alter von 43 bis 69 Jahren ist davon auszugehen, dass sie sogar die Ursache für eine zweifach erhöhte Mortalitätsrate von Patienten mit retinalem Venenverschluss sind (Cugati et al. 2007). Dabei ist die arterielle Hypertonie (32-70 \%) von größter Bedeutung, gefolgt von der Hyperlipidämie (30-60 \%), der koronaren Herzkrankheit (22-50 \%) und dem Diabetes mellitus (14-34 \%) (Feltgen 2008). Zusätzlich steigern weitere Faktoren wie Adipositas, Nikotin und hohes Lebensalter das Risiko (Cugati et al. 2007; Cheung et al. 2008; Klein 2008; O'Mahoney 2008; Yau et al. 2008; Wong und Scott 2010). Rheologische Besonderheiten wie eine erhöhte Viskosität, ein gesteigerter Hämatokrit, eine erhöhte Erythrozytenaggregation oder eine erniedrigte Verformbarkeit der Erythrozyten gelten vor allem als Kofaktoren und sind meist nicht die alleinige Ursache eines retinalen Venenverschlusses (Wiek et al. 1990; Hansen 2007). Von den hereditären Thrombophilien gelten nachweislich nur die Hyperhomozysteinämie und das Antiphospholipid-Antikörper-Syndrom als Risikofaktoren (Janssen et al. 2005; Fegan 2002; Ingerslev 1999). Bei jungen Patienten ohne Risikofaktoren ist es jedoch nach einem Verschlussereignis im Auge sinnvoll, nach Veränderungen der Blutgerinnung, einer erhöhten APCResistenz, einem Faktor-V-Leiden oder einem Faktor-XII-Mangel zu suchen, da diese Patienten meist kein kardiovaskuläres Risikoprofil aufweisen (Lerche et al. 2001; Kuhli-Hattenbach et al. 2009). Bei Patienten mit einem Hyperviskositätssyndrom, verursacht durch hämatologische Grunderkrankungen, die die Blutviskosität durch Vermehrung der Blutbestandteile erhöhen, sind immer beide Augen betroffen (Rodriguez und Eliott 2001; Dithmar et al. 2003). Als eine lokale Ursache eines retinalen Venenverschlusses ist, durch die Erhöhung des intraokularen Druckes, das Glaukom zu nennen (Hitchings und Spaeth 1976). Das Glaukom erhöht das 
Risiko eines retinalen Venenverschlusses um den Faktor 5 (Hansen 2007; Dithmar et al. 2003).

Die häufigsten Risikofaktoren für retinale Venenverschlüsse sind in der nachfolgenden Tabelle zusammengefasst.

\begin{tabular}{|ll|}
\hline Risikofaktoren & \\
kardiovaskuläre Erkrankungen & arterielle Hypertonie \\
(ZVV + VAV) & Diabetes mellitus \\
& Hyperlipidämie \\
& Übergewicht \\
& Rauchen \\
& \\
rheologische Besonderheiten & erhöhter Hämatokrit \\
(ZVV + VAV) & erhöhte Viskosität \\
& erhöhte Erythrozytenaggregation \\
& erniedrigte Verformbarkeit der Erythrozyten \\
& Hyperhomozysteinämie \\
Blutgerinnungsstörung & Antiphospholipid-AK-Syndrom \\
(ZVV + VAV) & erhöhte APC-Resistenz/FV-Leiden-Mutation \\
& erniedrigter Plasminogen-Aktivator-Inhibitor \\
& orale Kontrazeptiva \\
& Polyzythämia vera \\
lokale Risikofaktoren & Makroglobulinämie \\
(ZVV) & Myelom \\
Hyperviskositätssyndrom & Leukämie \\
(ZVV + VAV) & Glaukom \\
& Trauma \\
& retinale Vaskulitis \\
& Zentralarterienverschluss \\
& arteriovenöse Malformation \\
\hline & \\
& \\
& \\
& \\
& \\
&
\end{tabular}

Tabelle 1: Risikofaktoren für retinale Venenverschlüsse

(aus Feltgen 2008, S. 8 mit freundlicher Genehmigung) 


\subsubsection{Kardiovaskuläres Risiko}

Patienten mit einem kardiovaskulären Risikoprofil erleiden 3- bis 5-mal häufiger einen venösen Gefäßverschluss im Auge (Feltgen und Franko Zeitz 2014). Eine Metaanalyse aus Kanada zeigt, dass Patienten mit einem retinalem Venenverschluss eine erhöhte 10-Jahres-Inzidenz für kardiovaskuläre Erkrankungen aufweisen (Khan et al. 2013). Die FraminghamStudiengruppe untersuchte, welchen Einfluss kardiovaskuläre Risikofaktoren wie Alter, Geschlecht, HDL-Cholesterol, Gesamtcholesterin, systolischer Blutdruck und Rauchen auf die Entstehung von retinalen Venenverschlüssen haben (Khan et al. 2013). Mit Hilfe des Framingham-Risiko-Scores (FRS) lässt sich eine Risikoberechnung für kardiovaskuläre Erkrankungen innerhalb der nächsten 10 Jahre erheben und in folgende drei Gruppen einteilen:

- $\quad$ FRS $<10 \%$ niedriges kardiovaskuläres Risiko

- FRS $10 \%$ - 19 \% moderates kardiovaskuläres Risiko

- $\quad F R S \geq 20 \%$ hohes kardiovaskuläres Risiko

Das kardiovaskuläre Risiko für Patienten mit retinalem Venenverschluss lag im Mittel mit 10,1 \% über dem der kanadischen Normalbevölkerung (6 \%). Männliche Raucher hatten mit $28 \%$ das höchste Risiko zu erkranken, gefolgt von männlichen Nichtrauchern (17\%) und weiblichen Raucherinnen (11\%). Weibliche Nichtraucherinnen hatten ein niedriges Risiko von $8 \%$, was nahezu der Normalbevölkerung entsprach (Khan et al. 2013).

Neben der erhöhten Inzidenz für kardiovaskuläre Erkrankungen ist auch das Risiko für ein zerebrovaskuläres Ereignis bei Patienten mit retinalen Venenverschlüssen um das 1,5- bis 2-fache erhöht (Werther et al. 2011; Bertelsen et al. 2012). Erst kürzlich zeigte dies eine Studie an 87202 Patienten mit Vorhofflimmern, wovon 361 Patienten an einem retinalen Venenverschluss litten (Christiansen et al. 2013). Auch in einer Beobachtung von insgesamt 190 Patienten (45 Patienten mit venösem Gefäßverschluss im Auge) über acht 
Jahre erlitten Patienten mit retinalen Venenverschlüssen signifikant häufiger kardio- oder zerebrovaskuläre Ereignisse als Patienten ohne retinalen Gefäßverschluss (Di Capua et al. 2012).

Patienten mit einem Zentralvenenverschluss weisen zudem ein 1,5-fach erhöhtes Mortalitätsrisiko innerhalb von fünf Jahren auf (Bertelsen et al. 2014). Trotz dieser Daten ist die Qualität der publizierten Datensätze nicht immer ausreichend. Es handelt sich meist um retrospektive Analysen von Fallserien, die aber durchaus mit Kontrollgruppen verglichen wurden. Innerhalb des Untersuchungszeitraums waren die diagnostischen Methoden oft nicht konstant. Wünschenswert wäre eine prospektive Analyse mit einheitlichen diagnostischen Mitteln, wie sie in dieser Arbeit umgesetzt und in der Folge vorgestellt werden. 


\subsection{Zielsetzung}

Die FIND-AF-EYE-Studie ist eine interdisziplinäre, klinische Studie, die an der Universitätsmedizin Göttingen in Zusammenarbeit der Kliniken für Augenheilkunde, Kardiologie und Neurologie durchgeführt wurde. In der FINDAF-Studie (ISRCTN 46104198) konnte an Patienten mit einem Apoplex gezeigt werden, dass bei einer zerebralen Ischämie eine verlängerte Aufzeichnungsdauer mit einem 7-Tage-LZ-EKG gegenüber einem 24 h-EKG, signifikant mehr Patienten mit paroxysmalem Vorhofflimmern identifizierte. Deshalb wurde auch ein 7-Tage-LZ-EKG in der FIND-AF-EYE-Studie eingesetzt.

Ziel dieser Arbeit ist die systematische Abklärung kardiovaskulärer Risikofaktoren bei Patienten mit venösen Gefäßverschlüssen im Auge und darüber hinaus der Vergleich von Prävalenzen einzelner Risikofaktoren mit anderen zerebralen Ischämien (FIND-AF-Studie). Obwohl es sich dabei nicht um eine embolische Erkrankung handelt, weisen Patienten mit venösen retinalen Verschlüssen ebenfalls ein klares kardiovaskuläres Risikoprofil auf. Bisher gibt es kein einheitliches Diagnostikschema zur Ursachenabklärung eines retinalen Venenverschlusses. Diese Arbeit soll bei der Entscheidung, welche Routineuntersuchungen in Zukunft für venöse Gefäßverschlüsse im Auge als unverzichtbar anzusehen sind, helfen. 


\section{Patienten und Methoden}

\subsection{Studiendesign}

Die Datenerhebung erfolgte im Rahmen der FIND-AF-EYE-Studie. Es handelte sich um eine prospektive Evaluation kardiovaskulärer Risikofaktoren bei Patienten mit retinalen arteriellen oder venösen Gefäßverschlüssen. Die Studie hatte drei Arme, einen für Patienten mit einem arteriellen Verschluss, einen für Patienten mit venösem Verschluss der Netzhautgefäße und einen für Patienten mit einem Mischbild beider Verschlussformen. Eingeschlossen wurden alle arteriellen Verschlüsse (Zentralarterienverschluss, Arterienastverschluss, anteriore ischämische Optikusneuropathie), alle venösen Verschlüsse (Zentralvenenverschluss, Hemi-Zentralvenenverschluss, Venenastverschluss) und die Kombination aus beiden Verschlussformen (gemischte Verschlüsse). Im Zeitraum von Juni 2011 bis Juni 2012 wurden Patienten für die Studie an der Universitätsmedizin Göttingen rekrutiert.

Die stationäre Aufnahme der Verschlusspatienten erfolgte nicht nur im Rahmen der Studie. Auch bei Patienten, die nicht an der Studie teilnahmen, wurde nach möglichen Ursachen geforscht. Um in die Studie aufgenommen zu werden, mussten bestimmte Einschlusskriterien erfültt sein. Untersuchungszeitpunkte waren bei Einschluss, nach einem Monat und nach einem Jahr. Die Abbildung 7 zeigt eine schematische Darstellung des zeitlichen Ablaufes der Studie. Im Folgenden wird der Ablauf im Einzelnen erläutert. 


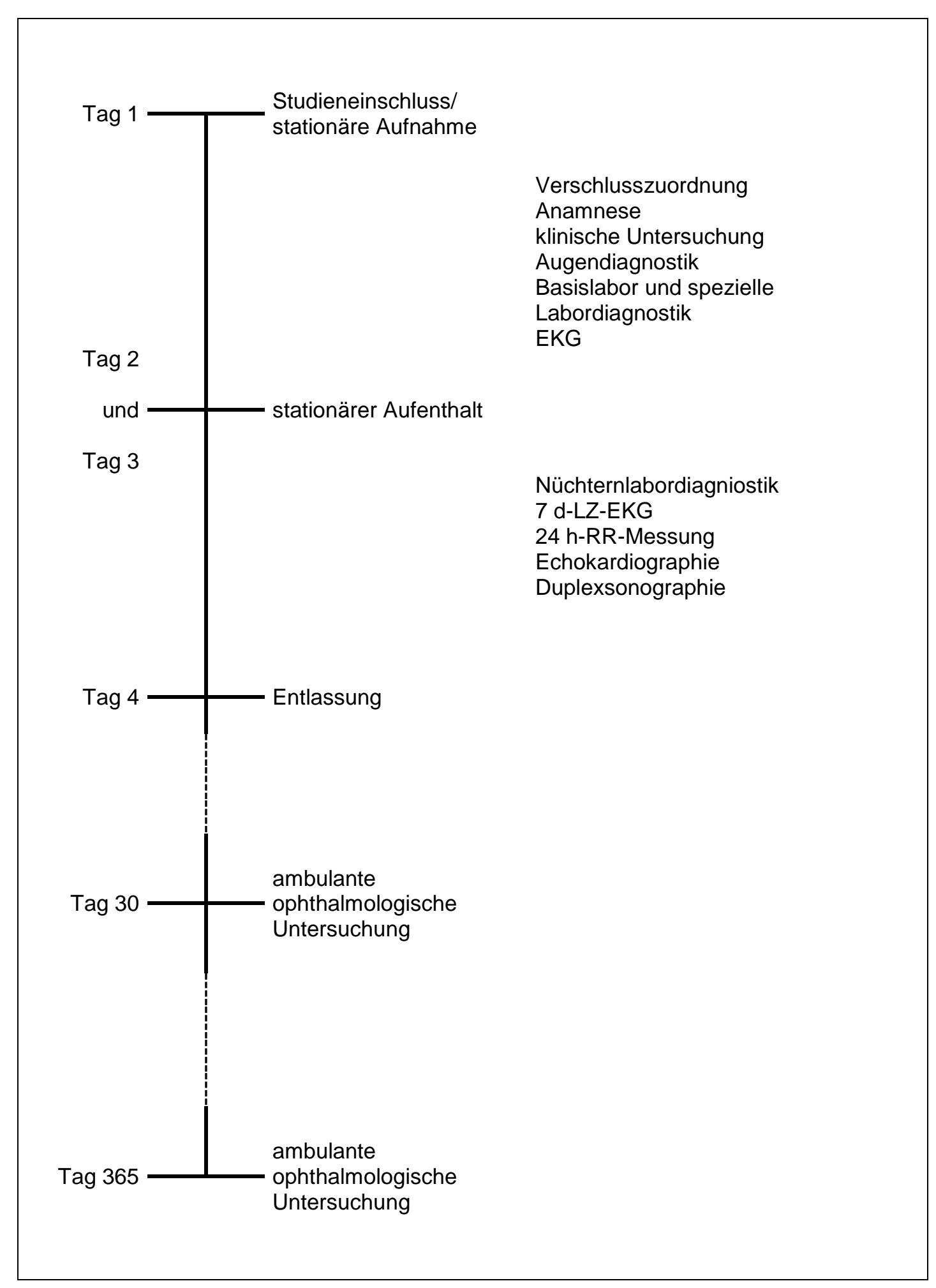

Abbildung 7: Zeitlicher Studienablauf 


\subsection{Patientenkollektiv}

\subsubsection{Einschlusskriterien}

Patienten im Alter von mindestens 18 Jahren mit einem Verschluss der Netzhautvene, einer Netzhautarterie oder einer versorgenden Arterie der Sehnerven wurden mit Vorliegen der schriftlichen Einverständniserklärung in die Studie eingeschlossen.

\subsubsection{Ausschlusskriterien}

Patienten mit fehlender Einwilligung oder unter dem 18. Lebensjahr wurden nicht in die Studie aufgenommen. Sie wurden anonymisiert mit Alter und Geschlecht in einer Screeningliste erfasst.

\subsection{Datenerfassung}

\subsubsection{Datenerfassung bei Aufnahme}

Mit Hilfe von Case Report Forms wurden die Daten systematisch erhoben. Je nach Verschluss erfolgte die Gruppenzuordnung durch den behandelnden Arzt in die 3 Hauptgruppen mit mehreren Untergruppen.

\begin{tabular}{|l|l|l|}
\hline arterielle Verschlüsse & venöse Verschlüsse & \multicolumn{2}{c|}{ gemischte } \\
\hline$\bullet$ & Zentralarterienverschlüse \\
\hline - Arterienastverschluss & $\bullet \quad$ Zentralvenenverschluss & $\bullet$ arteriovenös \\
$\bullet \quad$ nicht-arteriitische AION & $\bullet \quad$ Hemi-ZVV & \\
\hline
\end{tabular}

Tabelle 2: Gruppenzuordnung 


\subsubsection{Anamnese}

Von jedem Patienten wurde ein kardiovaskuläres Risikoprofil mit bekannten Risikofaktoren und Begleiterkrankungen erstellt. In der nachfolgenden Tabelle 3 sind die anamnestisch systematisch abgefragten Vorerkrankungen aufgelistet.

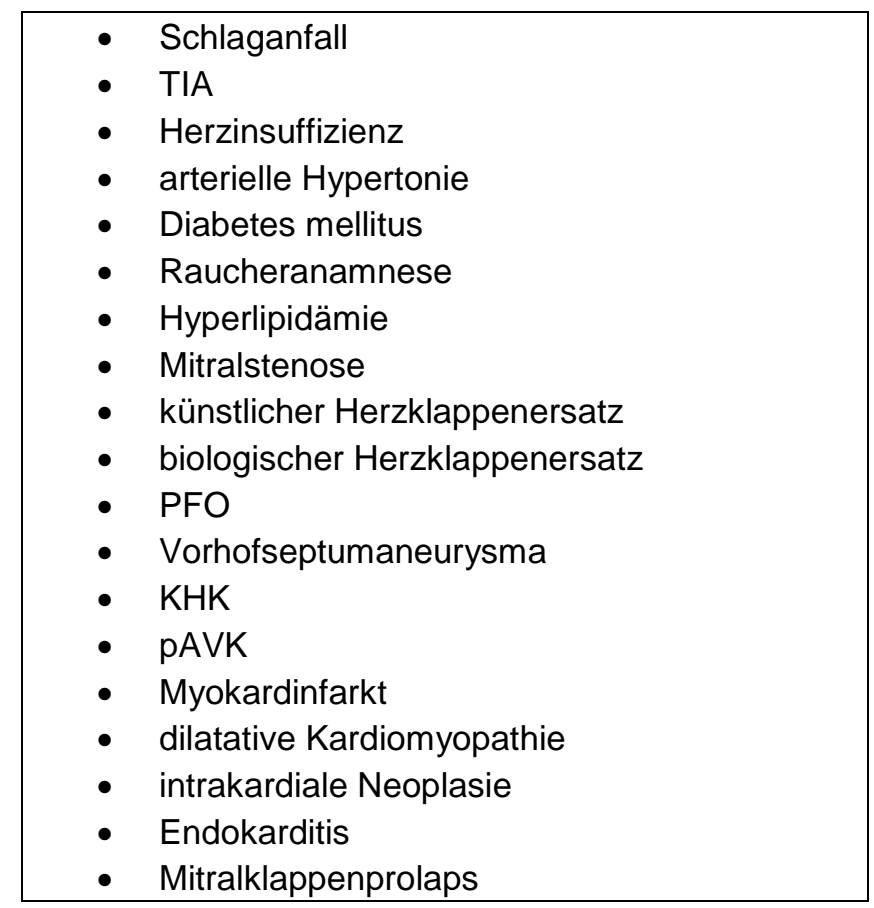

Tabelle 3: Anamnestisch systematisch abgefragte Vorerkrankungen 
Zusätzlich wurde ein kardiovaskuläres Medikamentenprofil erstellt. Medikamente der folgenden Gruppen wurden erfasst (s. Tabelle 4).

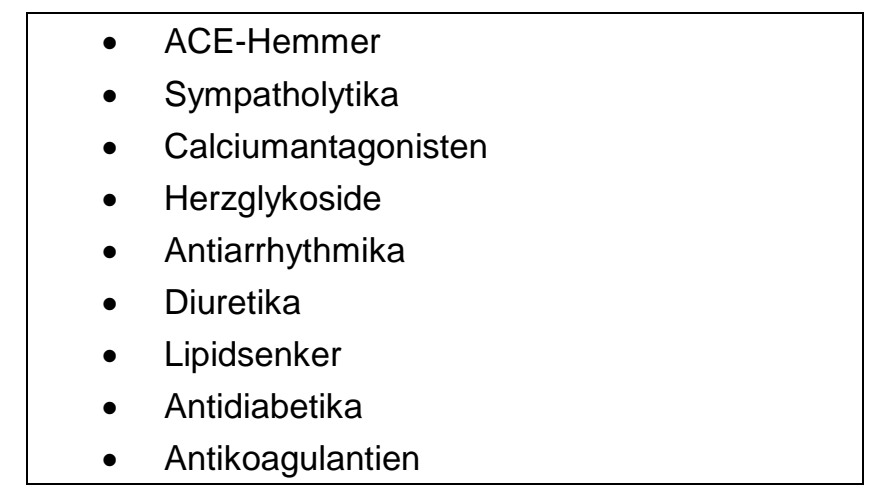

Tabelle 4: Medikamentenanamnese

Außerdem wurde nach ophthalmologischen Vorerkrankungen (s. Tabelle 5) und der regelmäßigen Augenmedikation gefragt.

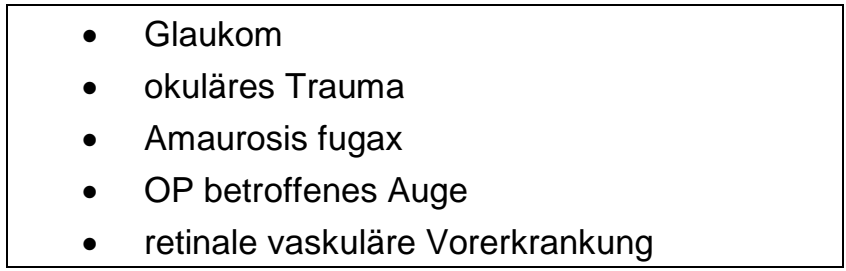

Tabelle 5: Ophthalmologische Vorerkrankungen

\subsubsection{Klinische Untersuchung}

Zu der klinischen Untersuchung zählten die in der Tabelle 6 aufgeführten Parameter.

\begin{tabular}{|clc|}
\hline$\bullet$ & Körpergröße & $\mathrm{cm}$ \\
$\bullet$ & Körpergewicht & $\mathrm{kg}$ \\
$\bullet$ & Blutdruckmessung & $\mathrm{mmHg}$ \\
$\bullet$ & Herzfrequenz & $1 / \mathrm{min}$ \\
\hline
\end{tabular}

Tabelle 6: Klinische Untersuchungsparameter 


\subsubsection{Basislabordiagnostik}

Die Basislabordiagnostik erfolgte am Aufnahmetag. Es umfasste die in der Tabelle 7 aufgeführten Messwerte. Die Nüchternlabordiagnostik (s. Tabelle 8) des Lipid- und Glukosestatus wurde am Folgetag erhoben.

\begin{tabular}{|l|}
\hline - INR \\
- HPTT \\
- Hämoglobin \\
- MCV \\
- Leukozokrit \\
- Thrombozyten \\
- Serumkreatinin \\
- Natrium \\
- Kalium \\
- Glukose \\
- TSP \\
- BSG \\
- Fibrinogen \\
- Harnsäure
\end{tabular}

Tabelle 7: Basislabor

\begin{tabular}{|l|}
\hline - Gesamtcholesterin \\
- LDL-Cholesterol \\
- TDL-Cholesterol \\
- Nriglyceride \\
- Güchternglukose \\
\hline
\end{tabular}

Tabelle 8: Nüchternlabordiagnostik 


\subsubsection{Spezielle Labordiagnostik}

Zum weiteren Verständnis der zugrundeliegenden Gefäßerkrankung wurden den Patienten in nicht nüchternem Zustand ca. $30 \mathrm{ml}$ Blut abgenommen. Eine Lithium-Heparin-Monovette, eine Serum-Monovette und eine EDTAMonovette wurden danach umgehend für 10 Minuten in einer EppendorfZentrifuge vom Typ 5702R zentrifugiert. Der Überstand der drei Monovetten wurde ab pipettiert und zusammen mit einer weiteren EDTA-Vollblutprobe bei $-80^{\circ} \mathrm{C}$ eingefroren. Die Blutproben sollen bei studienspezifischen Fragestellungen, die im Zusammenhang mit der Grunderkrankung bzw. der Therapie stehen, weiterhelfen. Ziel ist es, mit Hilfe eines Bluttests eine verbesserte Risikoanalyse für Gefäßverschlüsse im Auge zu schaffen.

\subsubsection{Augen-Diagnostik}

Die durchgeführte Augen-Diagnostik umfasste die Prüfung der Sehschärfe und des Augeninnendrucks beider Augen. In Einzelfällen wurde während des stationären Aufenthaltes ein Tagesdruckprofil über mehrere Tage erstellt. Individuell wurde auch über die Durchführung eines Augenfundusfotos, einer Angiographie der Netzhautgefäße und einer Netzhautdickemessung mittels einer optischen Kohärenztomographie (OCT) entschieden.

\subsubsection{Elektrokardiogramm}

Das Aufnahme-EKG bestand aus insgesamt 12 Ableitungen, drei bipolaren Extremitätenableitungen nach Einthoven, drei unipolaren Ableitungen nach Goldberger und sechs unipolaren Brustwandableitungen nach Wilson. Rhythmus, Frequenz, Leitungszeiten, Erregungsausbreitung und Erregungsrückbildung wurden beurteilt. 


\subsubsection{Datenerhebung während des stationären Aufenthaltes}

\subsubsection{7-Tage-Langzeit-EKG}

Bei fehlendem Vorhofflimmern im Aufnahme-EKG wurde ein 7-Tage-LangzeitEKG der Serie CardioMem® CM 3000 angelegt. In der folgenden Abbildung ist die Platzierung der fünf Elektroden auf dem Brustkorb dargestellt. Bei Entlassung wurden die Patienten in den Umgang mit dem Gerät eingewiesen und mit Wechselelektroden ausgestattet. Außerdem bekamen sie ein bereits adressiertes und frankiertes Päckchen zum Zurücksenden der Geräte mit nach Hause. Die Auswertung mit der Suche nach Vorhofflimmern erfolgte durch einen Kardiologen. Die Unterteilung erfolgte pro Tag in Gesamtdauer, Dauer der längsten Episode, Dauer der kürzesten Episode und durchschnittliche Episodendauer des Vorhofflimmerns.

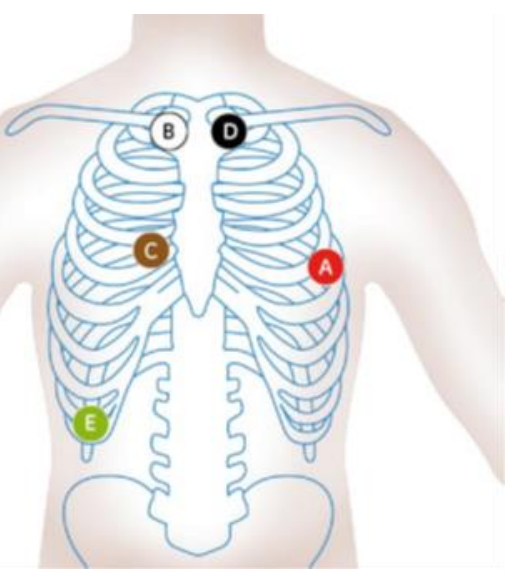

\begin{tabular}{lll} 
& \multicolumn{3}{c}{ Abbildung 8: Elektrodenplatzierung 7 d-LZ-EKG } \\
& Farbe & Position \\
A & $\bullet$ & 5. ICR, vordere Axillarlinie \\
B & $\circ$ & unterhalb der rechten Klavikula, medial zur mittleren Klavikularlinie \\
C & $\bullet$ & 4. ICR, rechter Sternalrand \\
D & $\bullet$ & unterhalb der linken Klavikula, medial zur mittleren Klavikularlinie \\
E & $\bullet$ & untere rechte Thoraxwand
\end{tabular}

(Anandic Medical Systems AG 2007) 


\subsubsection{Echokardiographie}

Während des stationären Aufenthaltes erhielten alle Patienten eine echokardiographische Untersuchung, die durch einen Kardiologen durchgeführt wurde. In einer transthorakalen Echokardiographie wurden mögliche Emboliequellen des Herzens und der Herzklappenstatus untersucht. Gegebenenfalls wurde zur weiteren Diagnostik eine transösophageale Echokardiographie durchgeführt. Dies lag im Ermessen des Kardiologen. Veränderungen der Herzklappen wurden in leicht- mittel- und schwergradig eingeteilt.

\subsubsection{Duplexsonographie}

Im stationären Verlauf wurden die Aa. carotides aller Patienten duplexsonographisch durch die Neurologen auf Plaques und Stenosen untersucht. Stenosegrade $>50 \%$ galten als Karotisstenose.

\subsubsection{24 h-Langzeitblutdruckmessung}

Jeder Patient erhielt nach der stationären Aufnahme eine Langzeitblutdruckmessung über 24 Stunden. Es wurde nach hypertonen systolischen und diastolischen Durchschnittswerten gesucht. Die Auswertung der Ergebnisse erfolgte durch einen Internisten. Systolische Durchschnittswerte $>140 \mathrm{mmHg}$ oder diastolische Durchschnittswerte $>90 \mathrm{mmHg}$ galten als Hypertonie.

\subsubsection{Datenerhebung bei Entlassung}

Der stationäre Aufenthalt dauerte im Durchschnitt 4 Tage. Bei der Entlassung der Patienten wurden Prognose- relevante Therapien im stationären Verlauf erfasst. Dazu zählten neben der Behandlung der Grunderkrankung des 
betroffenen Auges durch isovolämische Hämodilution, intravitreale Injektion, Laserbehandlung, Gabe von systemischen Steroiden, Thrombozytenaggregationshemmern und niedermolekularen Heparinen auch die Empfehlung der medikamentösen Therapie von kardiovaskulären Erkrankungen.

\subsubsection{Visite nach 1 Monat und 1 Jahr}

Die Patienten wurden nach einem Monat und nach einem Jahr nochmal zu einer ambulanten augenärztlichen Untersuchung in die Poliklinik der Augenheilkunde gebeten. Die ophthalmologische Diagnostik umfasste die Messung der Sehschärfe und des Augeninnendruckes des betroffenen Auges. Individuell wurde über weitere Untersuchungen entschieden.

Es erfolgte eine kurze Anamnese bezüglich Änderungen im kardiovaskulären Risikoprofil, neuer kardiovaskulärer Erkrankungen und in der medikamentösen Behandlung.

\subsection{Statistische Datenauswertung}

Die in der Studie erhobenen Daten sind als Mittelwert mit Standardabweichung oder als Anzahl mit Prozentzahl dargestellt. In einem weiteren Schritt wurden die Ergebnisse mit Ergebnissen eines bereits vorhandenen Datensatzes der FIND-AF-Studie verglichen. Die Analysen erfolgten mittels Chi-Quadrat Test nach Pearson. Das Signifikanzniveau wurde für $p \leq 0,05$ festgelegt.

Für die durchgeführten Analysen wurde das Programm STATISTICA Version 10 der Firma StatSoft $\circledast$ verwendet. Die Tabellen wurden mit Microsoft $₫$ Excel 2013 erstellt. 


\section{Ergebnisse}

\subsection{Patientenkollektiv}

Im Zeitraum von Juni 2011 bis Juni 2012 wurden insgesamt 101 Patienten in die FIND-AF-EYE-Studie eingeschlossen. Davon wurden 51 Patienten als arterielle, 41 Patienten als venöse und 9 Patienten als gemischte Verschlüsse klassifiziert (s. Abb. 9).
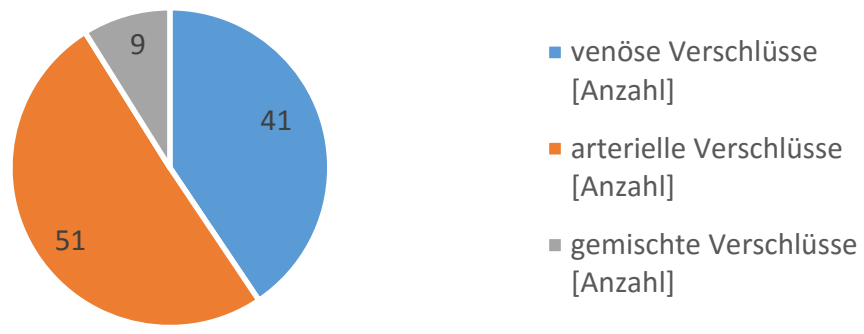

Abbildung 9: Verteilung der Gefäßverschlüsse

Von den 41 Patienten mit venösem Gefäßverschluss im Auge erlitten 26 Patienten einen ZVV, 11 Patienten einen VAV und 4 Patienten einen HZVV (s. Abb. 10).
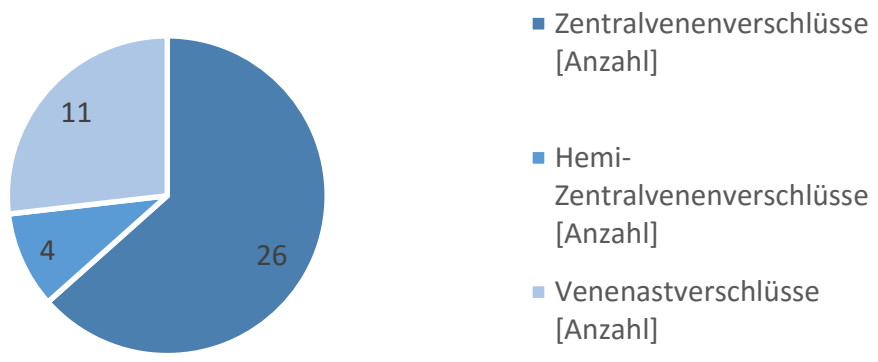

Abbildung 10: Verteilung der venösen Gefäßverschlüsse 
Bei 22 Patienten (54 \%) war das rechte Auge betroffen, bei 19 Patienten (46\%) das linke Auge (s. Abb. 11).

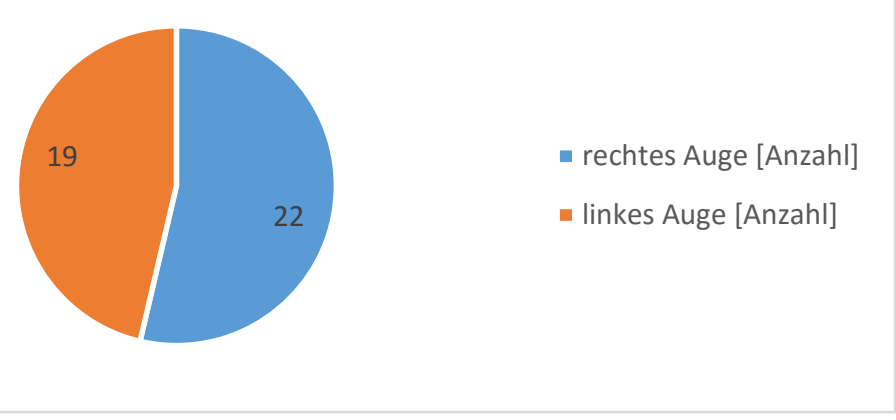

Abbildung 11: Verteilung der betroffenen Augen

17 Patienten (42 \%) waren Frauen und 24 Patienten (59 \%) Männer (s. Abb. 13). Der Altersdurchschnitt lag bei $65 \pm 13,3$ [MW \pm SD] Jahren (s. Abb. 14). Der jüngste Patient war 35 Jahre, der älteste Patient 86 Jahre alt.

Im Mittel betrug der BMI $29 \pm 6,9$ kg/m² (s. Abb. 15). Bei 21 Patienten (51 \%) war der BMI > $25 \mathrm{~kg} / \mathrm{m}^{2}$ und sie waren somit übergewichtig. 9 Patienten (22\%) waren adipös mit einem $\mathrm{BMI}>30 \mathrm{~kg} / \mathrm{m}^{2}$.

\subsubsection{Klinische Charakteristika}

Die Tabelle 9 zeigt die klinischen Charakteristika der Patienten mit retinalen Venenverschlüssen (FIND-AF-EYE-Studie) und die der Patienten mit zerebraler Ischämie oder TIA (FIND-AF-Studie) im Vergleich. Im Folgenden beschreibe ich zuerst die Ergebnisse der Patienten mit venösen Gefäßverschlüssen im Auge (FIND-AF-EYE-Studie) bevor ich in einem nächsten Schritt einzelne Prävalenzen der beiden Patientenkollektive vergleiche. 


\begin{tabular}{|c|c|c|c|c|c|}
\hline & $\mathrm{n}$ & $\begin{array}{c}\text { FIND-AF-EYE- } \\
\text { Studie } \\
\text { Venöse } \\
\text { Verschlüsse } \\
\text { MW } \pm \text { SD } \\
\text { Anzahl (\%) }\end{array}$ & $\mathrm{n}$ & $\begin{array}{c}\text { FIND-AF-Studie } \\
\text { Zerebrale Ischämie/ } \\
\text { TIA } \\
\text { MW } \pm \text { SD } \\
\text { Anzahl (\%) }\end{array}$ & $\begin{array}{c}p- \\
\text { Wert }\end{array}$ \\
\hline \multicolumn{6}{|l|}{ Demographische Daten } \\
\hline \multicolumn{6}{|l|}{ Geschlecht } \\
\hline weiblich & \multirow{2}{*}{41} & $17(41,5 \%)$ & \multirow{2}{*}{272} & $121(44,5 \%)$ & \multirow{2}{*}{0,72} \\
\hline männlich & & $24(58,5 \%)$ & & $151(55,5 \%)$ & \\
\hline Alter (y) & 41 & $65 \pm 13,3$ & 272 & $70 \pm 12$ & 0,02 \\
\hline $\mathrm{BMI}\left(\mathrm{kg} / \mathrm{m}^{2}\right)$ & 41 & $29 \pm 6,9$ & 240 & $27,7 \pm 5,7$ & 0,26 \\
\hline \multicolumn{6}{|l|}{ betroffenes Auge } \\
\hline rechts & \multirow{2}{*}{41} & $22(53,7 \%)$ & & & \\
\hline links & & $19(46,3 \%)$ & & & \\
\hline \multicolumn{6}{|c|}{ Risikofaktoren und Vorerkrankungen } \\
\hline systolischer RR (mmHg) & 40 & $149 \pm 26,5$ & 271 & $144 \pm 25$ & 0,29 \\
\hline diastolischer RR (mmHg) & 39 & $86 \pm 15,9$ & 271 & $80 \pm 13,9$ & 0,03 \\
\hline Herzfrequenz $(1 / \mathrm{min})$ & 37 & $75 \pm 14,1$ & 272 & $73 \pm 14,7$ & 0,52 \\
\hline bekannter Schlaganfall & 41 & $1(2,4 \%)$ & 272 & $42(15,4 \%)$ & 0,02 \\
\hline bekannte TIA & 39 & $0(0 \%)$ & 272 & $26(9,6 \%)$ & 0,04 \\
\hline bekannte Herzinsuffizienz & 40 & $2(5 \%)$ & 272 & $19(7 \%)$ & 0,64 \\
\hline $\begin{array}{c}\text { arterielle Hypertonie } \\
\text { gesamt } \\
\text { arterielle Hypertonie } \\
\text { neu diagnostizierte } \\
\text { arterielle Hypertonie } \\
\end{array}$ & $\begin{array}{r}41 \\
41 \\
18\end{array}$ & $\begin{array}{c}31(75,6 \%) \\
27(65,9 \%) \\
4(22,2 \%)\end{array}$ & 272 & $201(73,9 \%)$ & 0,32 \\
\hline $\begin{array}{c}\text { Diabetes mellitus gesamt } \\
\text { Diabetes mellitus } \\
\text { neu diagnostizierter } \\
\text { Diabetes mellitus }\end{array}$ & $\begin{array}{l}41 \\
41 \\
29\end{array}$ & $\begin{array}{l}8(19,5 \%) \\
6(14,6 \%) \\
2(6,9 \%)\end{array}$ & 272 & $67(24,3 \%)$ & 0,11 \\
\hline (ehemalige) Raucher & 39 & $21(53,8 \%)$ & 272 & $101(37,1 \%)$ & 0,05 \\
\hline $\begin{array}{c}\text { Hyperlipidämie gesamt } \\
\text { Hyperlipidämie } \\
\text { neu diganostizierte } \\
\text { Hyperlipidämie }\end{array}$ & $\begin{array}{l}39 \\
39 \\
25\end{array}$ & $\begin{array}{c}14(35,9 \%) \\
11(28,2 \%) \\
3(12 \%)\end{array}$ & 272 & $93(34,2 \%)$ & 0,34 \\
\hline
\end{tabular}




\begin{tabular}{|c|c|c|c|c|c|}
\hline & $\mathrm{n}$ & $\begin{array}{c}\text { FIND-AF-EYE- } \\
\text { Studie } \\
\text { Venöse } \\
\text { Verschlüsse } \\
\text { MW } \pm \text { SD } \\
\text { Anzahl (\%) } \\
\end{array}$ & $\mathrm{n}$ & $\begin{array}{c}\text { FIND-AF-Studie } \\
\text { Zerebrale Ischämie/ } \\
\text { TIA } \\
\text { MW } \pm \text { SD } \\
\text { Anzahl (\%) }\end{array}$ & $\begin{array}{c}p- \\
\text { Wert }\end{array}$ \\
\hline bekannte KHK & 41 & $2(4,9 \%)$ & 272 & $43(15,8 \%)$ & 0,06 \\
\hline bekannte pAVK & 39 & $1(2,6 \%)$ & 272 & $8(2,9 \%)$ & 0,9 \\
\hline Vorhofflimmern gesamt & 41 & $8(19,5 \%)$ & 272 & $72(26,4 \%)$ & 0,31 \\
\hline $\begin{array}{l}\text { Vorhofflimmern } \\
\text { neu diagnostiziertes } \\
\text { Vorhofflimmern }\end{array}$ & $\begin{array}{l}41 \\
34 \\
\end{array}$ & $\begin{array}{c}5(12,2 \%) \\
3(8,8 \%)\end{array}$ & $\begin{array}{l}272 \\
225 \\
\end{array}$ & $\begin{array}{l}43(15,8 \%) \\
29(12,9 \%)\end{array}$ & $\begin{array}{l}0,52 \\
0,46\end{array}$ \\
\hline Herzklappenveränderungen & 33 & $7(21,2 \%)$ & 246 & $61(24,8 \%)$ & 0,65 \\
\hline Karotisstenose & 35 & $2(5,7 \%)$ & 254 & $41(16,1 \%)$ & 0,03 \\
\hline \multicolumn{6}{|l|}{ Labor } \\
\hline Kreatinin (mg/dL) & 37 & $1,1 \pm 0,6$ & 271 & $1,02 \pm 0,56$ & 0,32 \\
\hline Glukose (mg/dL) & 33 & $125,1 \pm 46,5$ & 254 & $129 \pm 49$ & 0,67 \\
\hline Gesamtcholesterin (mg/dL) & 38 & $193,4 \pm 45,6$ & 257 & $194 \pm 47$ & 0,96 \\
\hline LDL-Cholesterin (mg/dL) & 36 & $118,6 \pm 45,7$ & 258 & $128 \pm 39$ & 0,27 \\
\hline HDL-Cholesterin (mg/dL) & 37 & $55,1 \pm 14,6$ & 258 & $50 \pm 16$ & 0,08 \\
\hline Triglyceride (mg/dL) & 38 & $136,9 \pm 51,2$ & 256 & $128 \pm 63$ & 0,36 \\
\hline
\end{tabular}

Tabelle 9: Klinische Charakteristika - Gruppen im Vergleich 


\subsubsection{Risikofaktoren und Vorerkrankungen}

Der systolische Blutdruck bei Aufnahme betrug im Mittel 149 \pm 26,5 [MW \pm SD] $\mathrm{mmHg}$, der diastolische Wert lag bei $86 \pm 15,9 \mathrm{mmHg}$. Bei 27 Patienten (66\%) war in der Vorgeschichte ein arterieller Hypertonus, bei 6 Patienten (15\%) ein Diabetes mellitus bekannt. 11 Patienten (28\%) gaben in der Anamnese eine Hyperlipidämie an. Bei Aufnahme war bereits bei 5 Patienten (12\%) ein Vorhofflimmern bekannt. Eine Raucheranamnese hatten 21 von 39 Patienten (54 \%). Davon waren 5 Patienten (24\%) Frauen und 16 Patienten (76 \%) Männer. Unter den 21 Patienten gab es 12 (31\%) ehemalige und 9 (23\%) aktive Raucher. Einen Myokardinfarkt erlitten zu haben, gaben 3 von 41 Patienten ( $7 \%$ ) an, eine Herzinsuffizienz hatten 2 von 40 Patienten ( $5 \%$ ), eine KHK 2 von 41 Patienten (5\%), eine pAVK 1 von 39 Patienten (3\%) und einen Schlaganfall 1 von 41 Patienten (2\%). Eine TIA und anamnestisch erhobene kardiale Vorerkrankungen wie Mitralstenosen, künstlicher oder biologischer Herzklappenersatz, PFO, Vorhofseptumaneurysma, dilatative Kardiomyopathie, intrakardiale Neoplasie, Endokarditis oder Mitralklappenprolaps lag bei keinem der Patienten vor. Die genannten Risikofaktoren und Vorerkrankungen sind in Abbildung 12 dargestellt.

\subsubsection{Neu diagnostizierte Risikofaktoren}

Bei 4 Patienten (22 \%) konnten wir durch eine 24-Stunden-Blutdruckmessung eine arterielle Hypertonie neu diagnostizieren. Durch die Bestimmung von Nüchternglukose- und HbA1c-Werten wurde bei 2 weiteren Patienten (7\%) ein Diabetes mellitus neu diagnostiziert. Bei 3 Patienten (12\%) wurde durch eine Nüchternblutprobe laborchemisch eine Hyperlipidämie neu diagnostiziert. Ein 7-Tage-Langzeit-EKG wurde bei 34 von den 36 verbliebenen Patienten angelegt und bei weiteren 3 Patienten $(9 \%)$ konnte im Verlauf ein 
paroxysmales Vorhofflimmern neu diagnostiziert werden. Eine Echokardiographie wurde bei 33 Patienten durchgeführt. Relevante Herzklappenveränderung, darunter fallen mittel- bis schwergradige Veränderungen der Herzklappen, konnten bei 7 Patienten (21\%) diagnostiziert werden. In einer Duplexsonographie wurde bei 2 von 35 Patienten (6\%) eine > 50 \%-Karotisstenose diagnostiziert. Die neu diagnostizierten Risikofaktoren sind in Abbildung 12 dargestellt. 


\subsubsection{Bekannte und neu diagnostizierte Risikofaktoren}

Insgesamt litten 31 Patienten (76 \%) an einem arteriellen Hypertonus, 8 Patienten (20\%) an einen Diabetes mellitus, 14 Patienten (36\%) an einer Hyperlipidämie und 8 Patienten (20\%) an einem Vorhofflimmern. In folgender Abbildung sind die bekannten und neu diagnostizierten Risikofaktoren graphisch dargestellt.

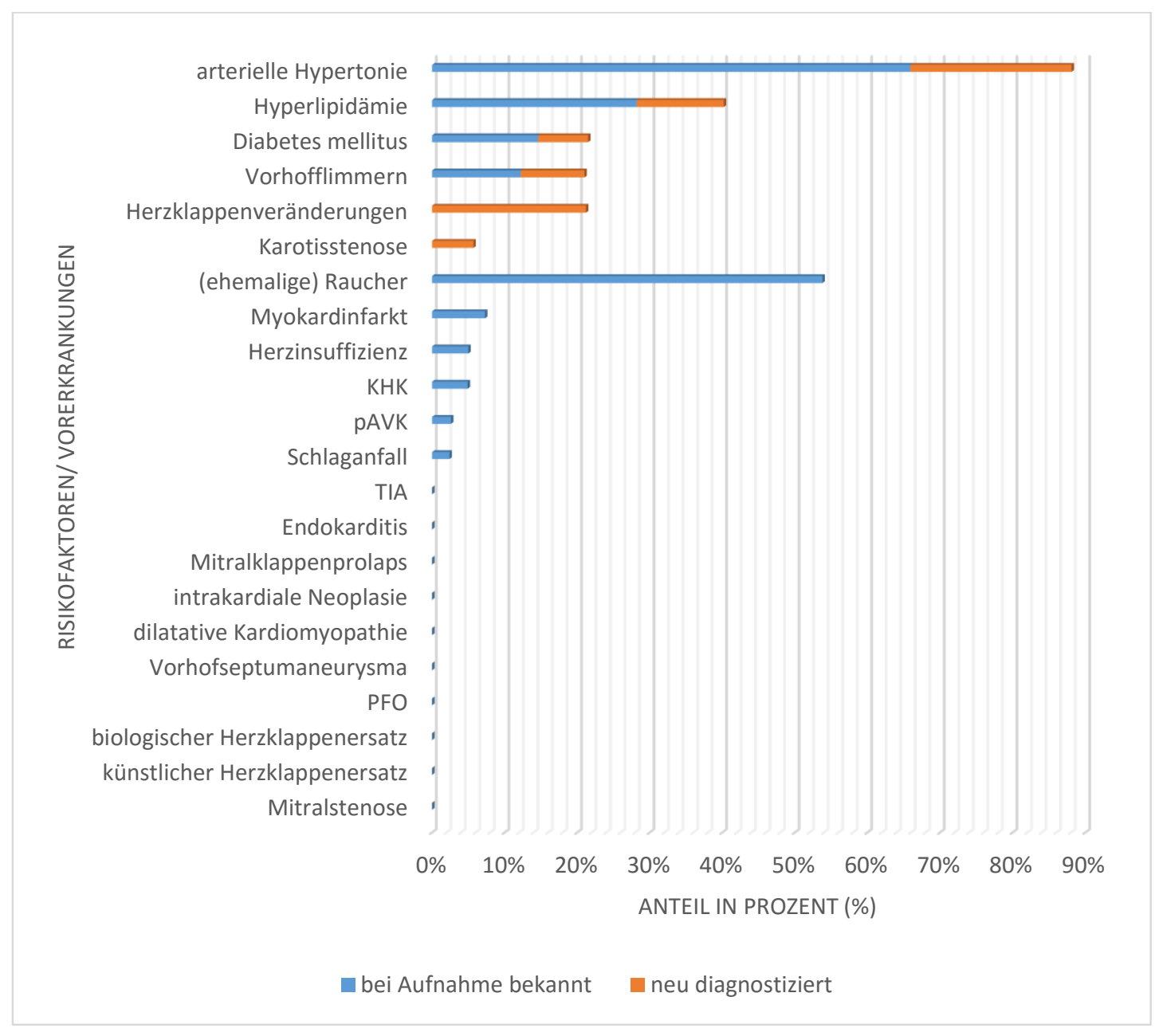

Abbildung 12: Bekannte und neu diagnostizierte Risikofaktoren 


\subsection{Vergleich einzelner Prävalenzen von Risikofaktoren bei Patienten mit anderen zerebralen Ischämien}

Im Vergleich des Patientenkollektivs liegt eine gleiche Geschlechterverteilung von Patienten mit Gefäßverschlüssen im Auge (FIND-AF-EYE-Studie) und Patienten mit zerebraler Ischämie vor (FIND-AF-Studie) (s. Abb. 13).

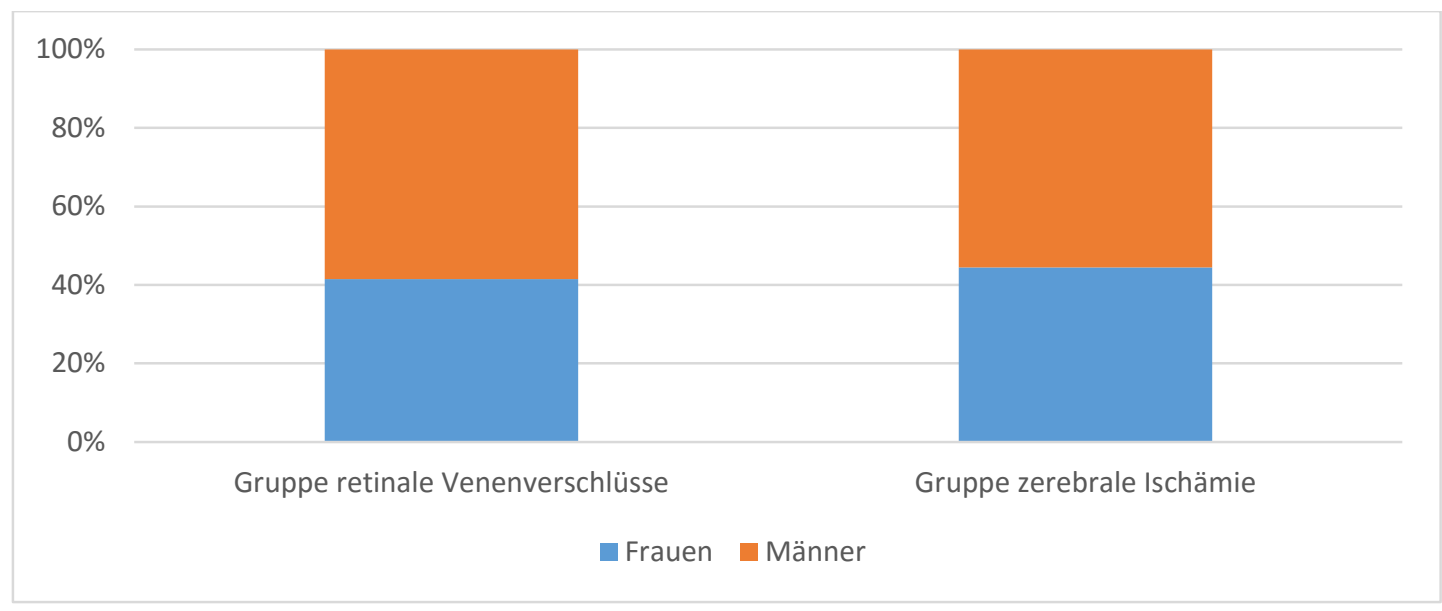

Abbildung 13: Geschlechterverteilung - Gruppen im Vergleich 
Beim Alter gab es einen signifikanten Unterschied (s. Abb. 14). Die Patienten mit retinalem Gefäßverschluss waren im Mittel mit $65 \pm$ 13,3 Jahren [MW \pm SD] 5 Jahre jünger als die Patienten mit zerebraler Ischämie ( $70 \pm 12$ Jahre).

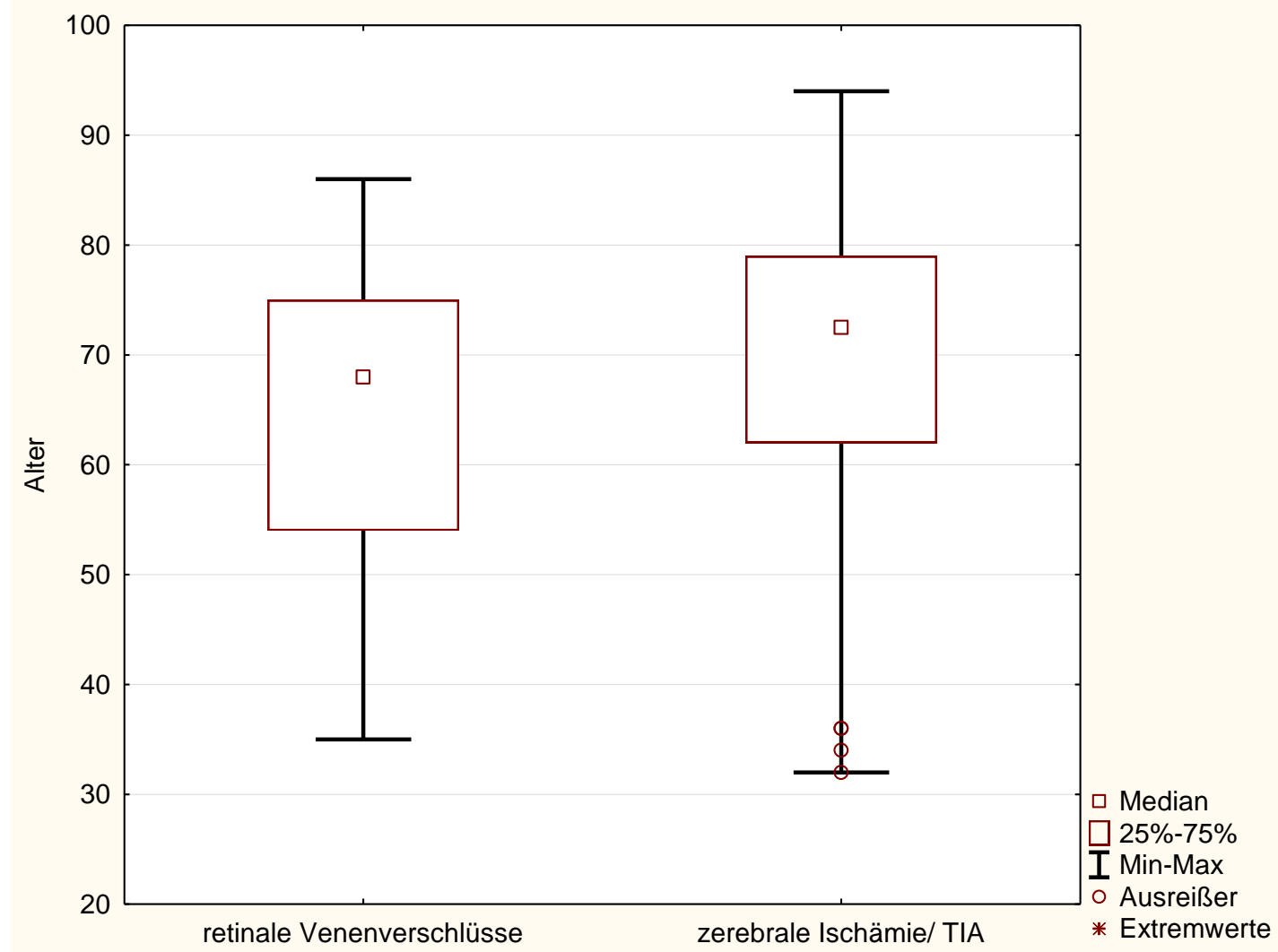

Abbildung 14: Boxplot Alter - Gruppen im Vergleich 
Wie in Abbildung 15 dargestellt, waren beide Gruppen übergewichtig. Die Patienten mit zerebraler Ischämie waren mit einem BMI von $27,7 \pm 5,7 \mathrm{~kg} / \mathrm{m}^{2}$ jedoch etwas schlanker als die Patienten mit Gefäßverschluss im Auge. Der BMI lag dort bei $29 \pm 6,9 \mathrm{~kg} / \mathrm{m}^{2}$.

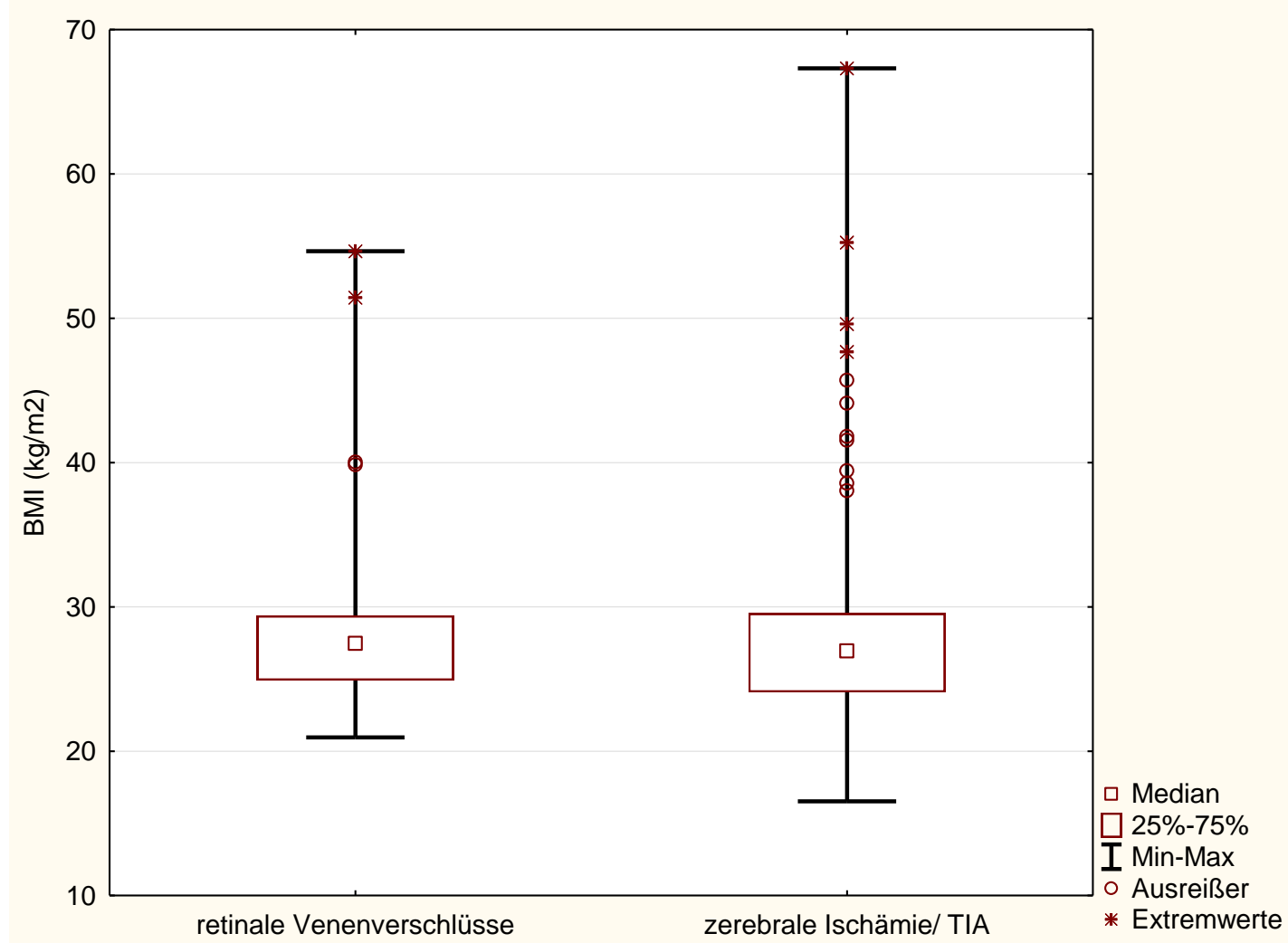

Abbildung 15: Boxplot BMI - Gruppen im Vergleich 
Bei den Risikofaktoren und Vorerkrankungen fiel folgendes auf. Der systolische Blutdruck bei Aufnahme war bei den Patienten mit retinalem Gefäßverschluss mit $149 \pm 26,5 \mathrm{mmHg}$ etwas höher als bei den Patienten mit zerebraler Ischämie $(144 \pm 25 \mathrm{mmHg})$. Beiden Gruppen wiesen leicht hypertone Blutdruckwerte auf (s. Abb. 16).

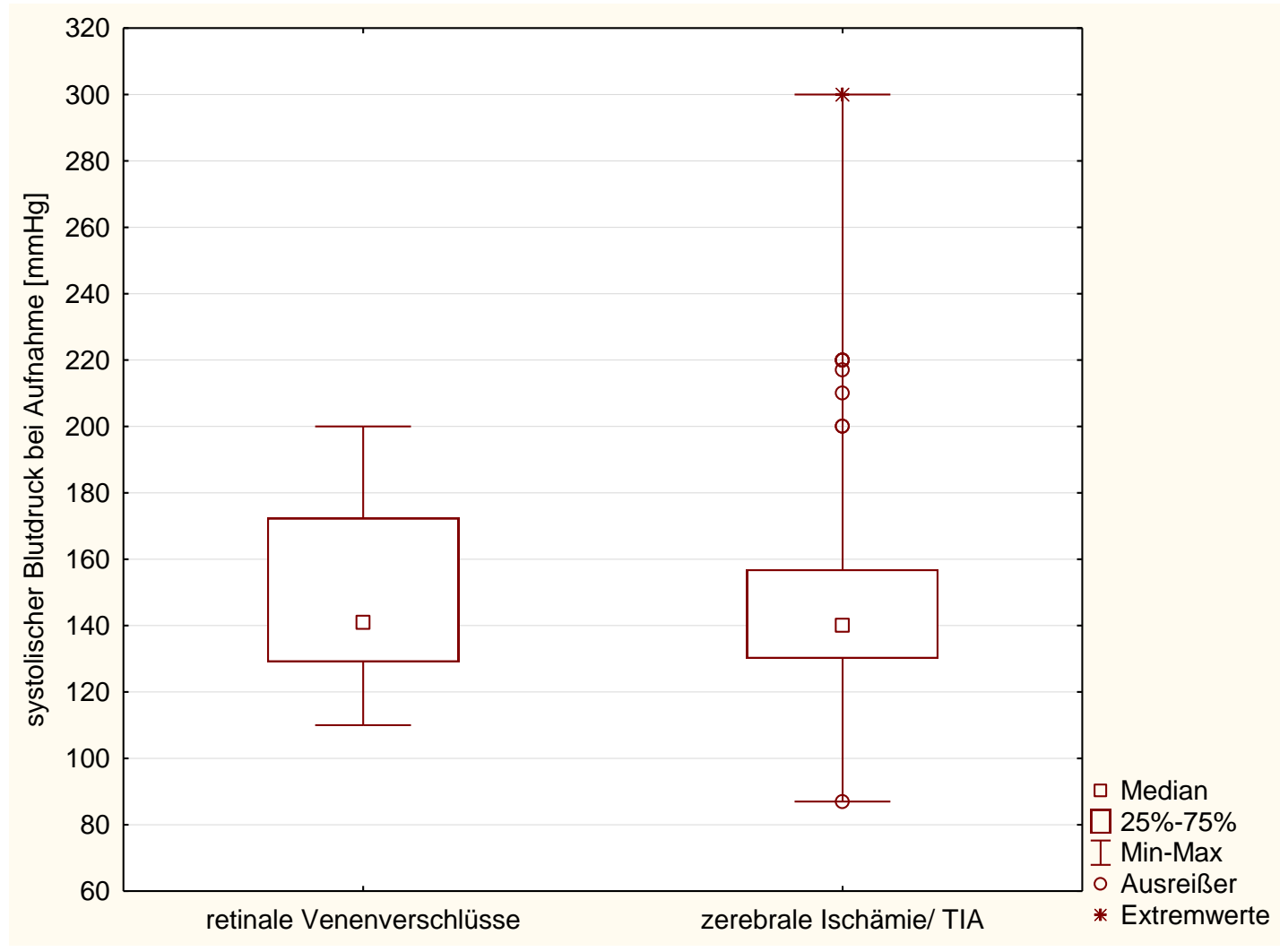

Abbildung 16: Boxplot systolischer Blutdruck - Gruppen im Vergleich 
Die diastolischen Blutdruckwerte lagen im Normbereich. Es gab jedoch einen signifikanten Unterschied. Patienten aus unserer Studie hatten mit $86 \pm 15,9 \mathrm{mmHg}$ einen höheren diastolischen Blutdruckwert im Vergleich zu Patienten aus der Gruppe der zerebralen Ischämien $(80 \pm 13,9 \mathrm{mmHg})$ (s. Abb. 17).

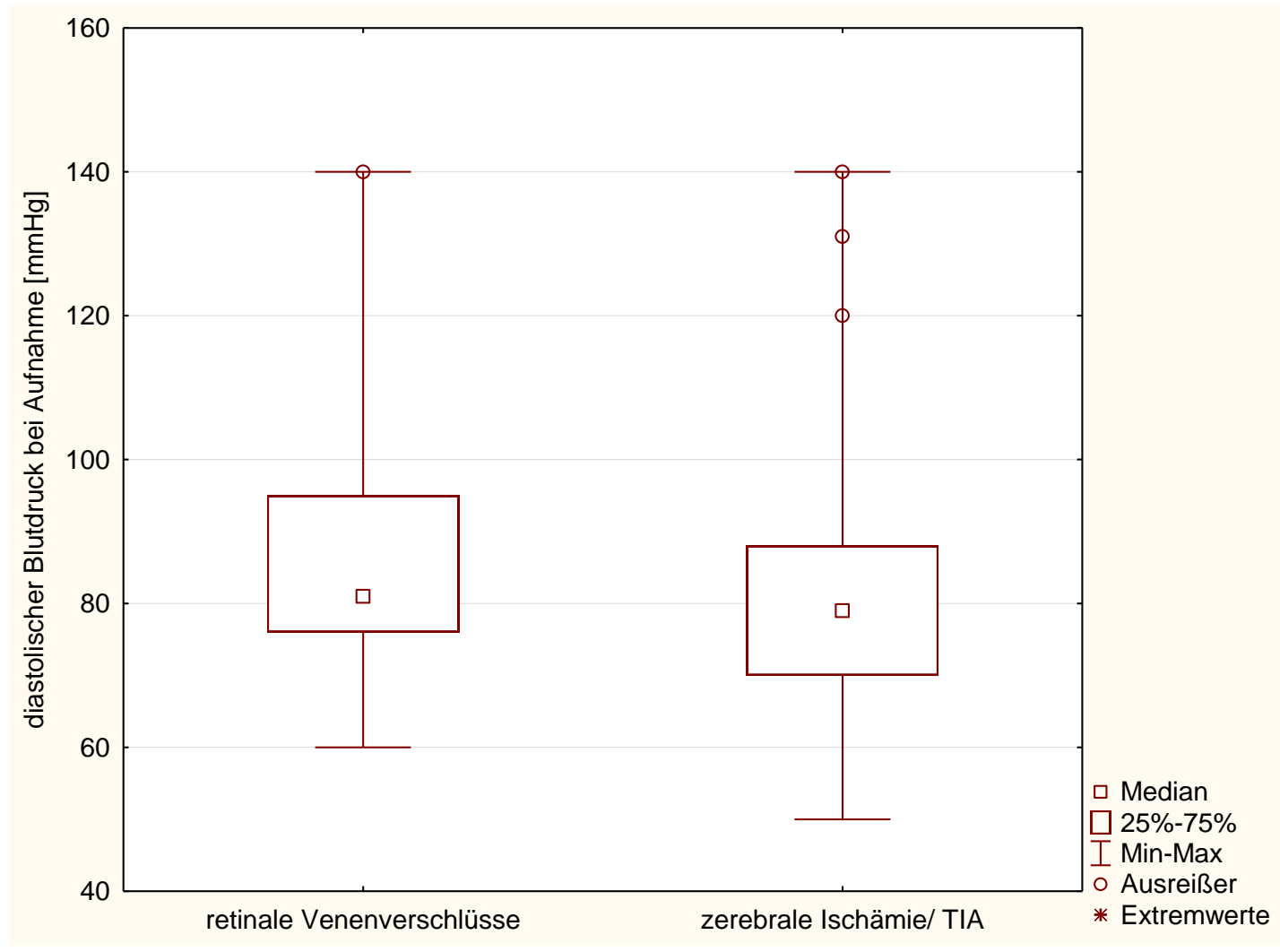

Abbildung 17: Boxplot diastolischer Blutdruck - Gruppen im Vergleich 
Die Abbildung 18 zeigt die Herzfrequenz bei Aufnahme, die bei beiden Gruppen im mittleren Normbereich lag.

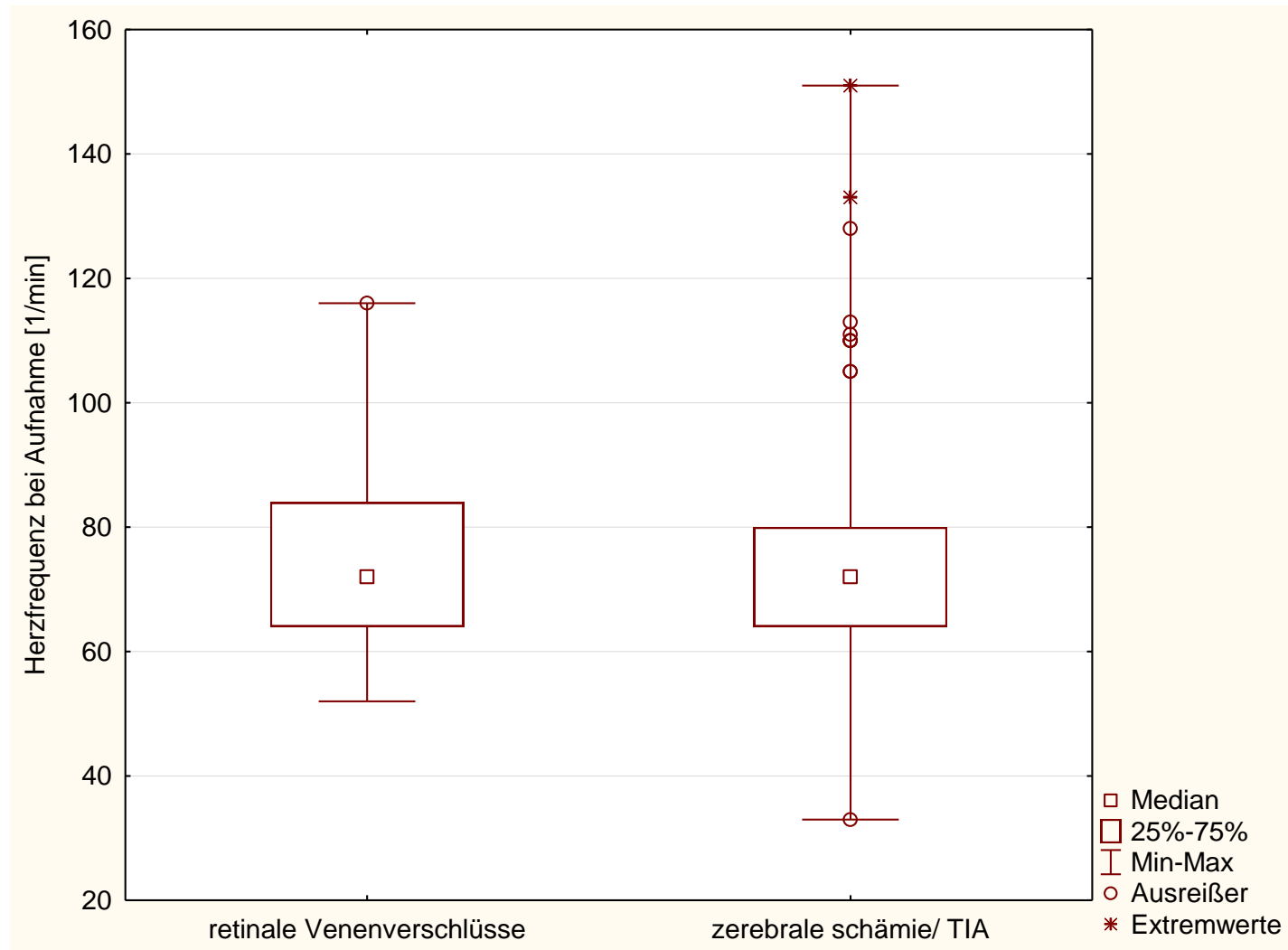

Abbildung 18: Boxplot Herzfrequenz - Gruppen im Vergleich 
Patienten mit zerebraler Ischämie erlitten signifikant häufiger in ihrer Vorgeschichte schon einmal einen Schlaganfall $(p=0,02)$ (s. Abb. 19) oder eine $\operatorname{TIA}(p=0,04)(s$. Abb.20).

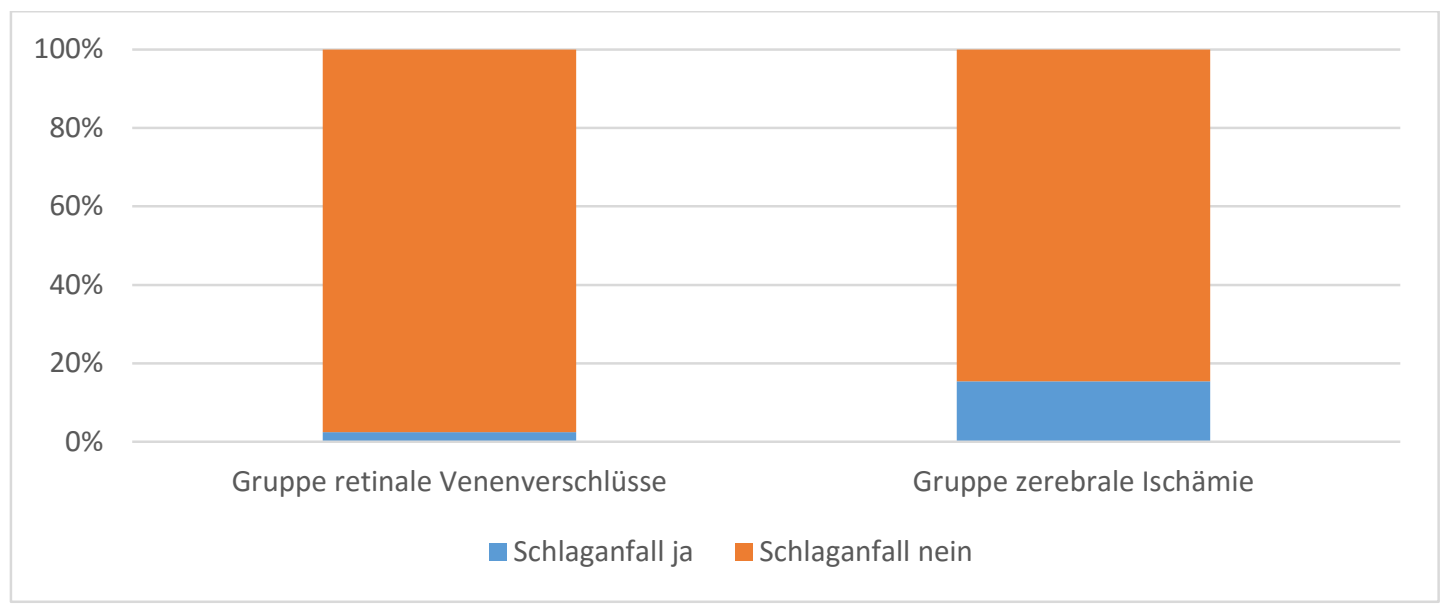

Abbildung 19: Schlaganfall - Gruppen im Vergleich

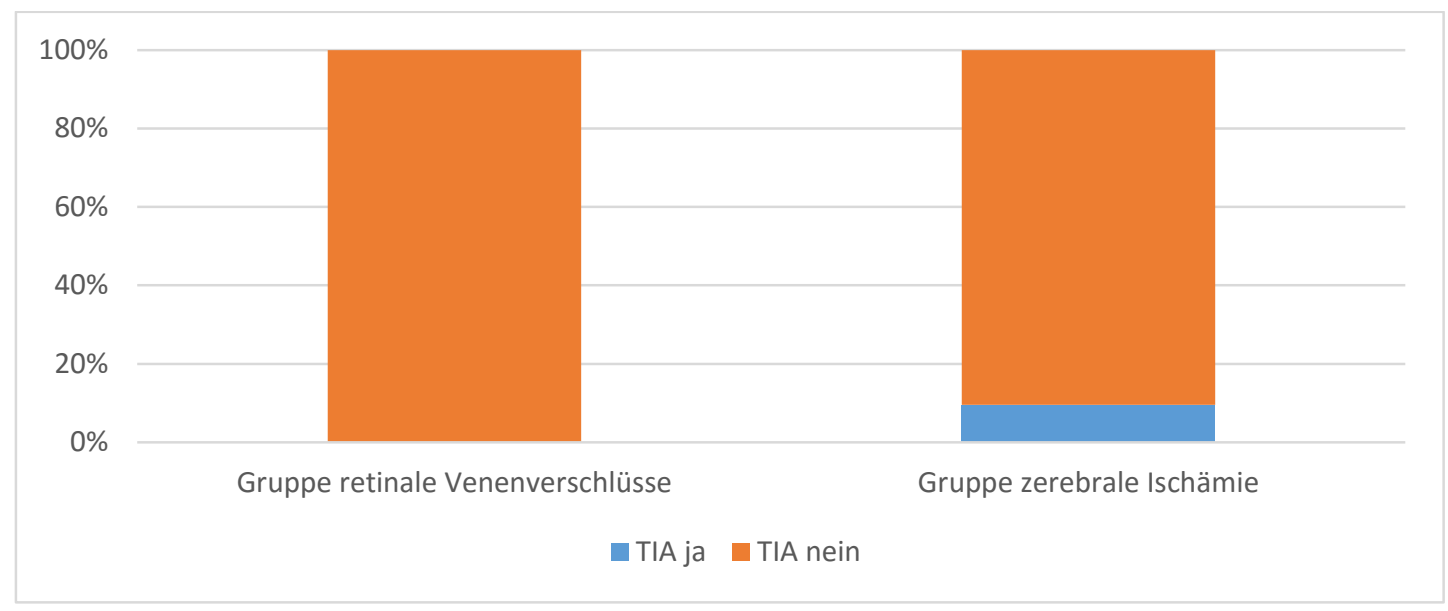

Abbildung 20: TIA - Gruppen im Vergleich 
Auch beim Rauchen gab es einen signifikanten Unterschied. Wie in Abbildung 21 dargestellt, rauchen mehr als die Hälfte der Patienten mit Gefäßverschluss im Auge (54 \%) oder haben früher geraucht, wohingegen nur etwas mehr als ein Drittel der Patienten mit zerebraler Ischämie (37 \%) eine Raucheranamnese angegeben haben $(p=0,05)$.

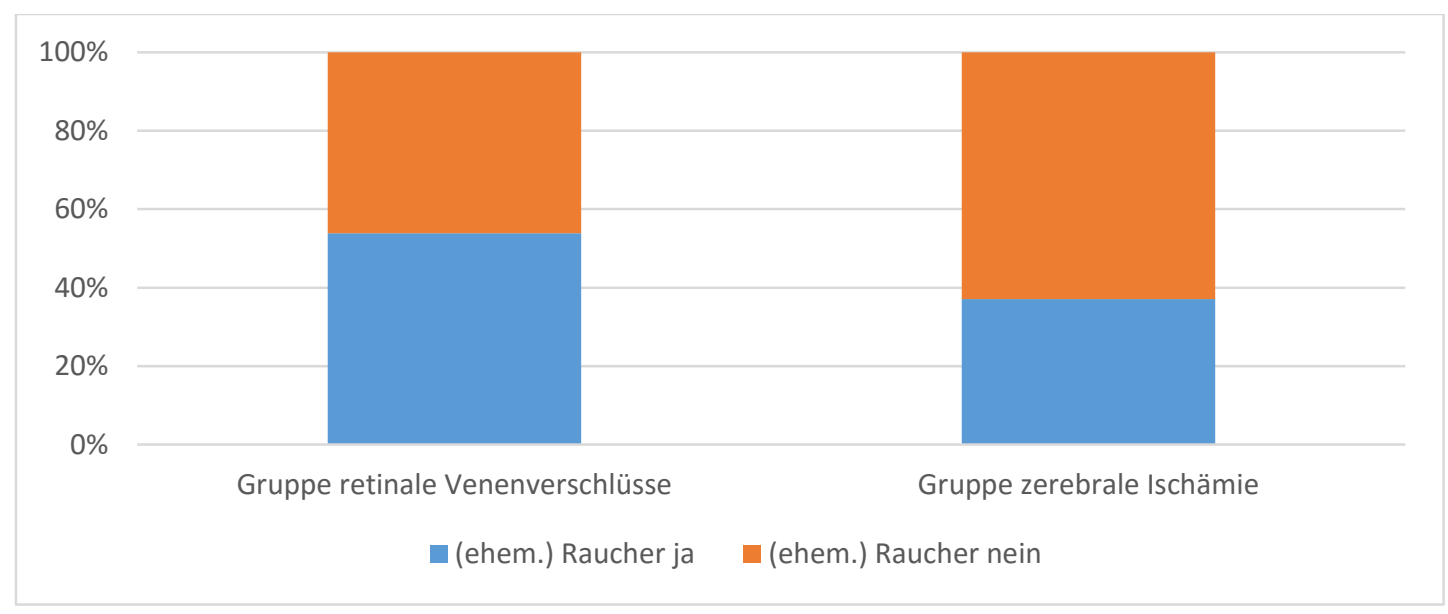

Abbildung 21: Raucheranamnese - Gruppen im Vergleich 
Kardiovaskuläre Risikofaktoren wie eine arterielle Hypertonie, ein Diabetes mellitus, eine Hyperlipidämie, ein Vorhofflimmern sowie relevante Herzklappenveränderungen waren in beiden Patientenkollektiven ähnlich häufig vertreten. Patienten mit zerebraler Ischämie litten jedoch signifikant häufiger unter einer > 50 \%-Karotisstenose als Patienten mit retinalem Venenverschluss $(p=0,03)$. Laborchemisch zeigten sich keine signifikanten Unterschiede.

Die Abbildung 22 zeigt den prozentualen Anteil der Patienten mit arterieller Hypertonie bei Aufnahme. In der Gruppe der retinalen Venenverschlüsse waren es $66 \%$ und in der Gruppe der zerebralen Ischämie 74 \% der Patienten.

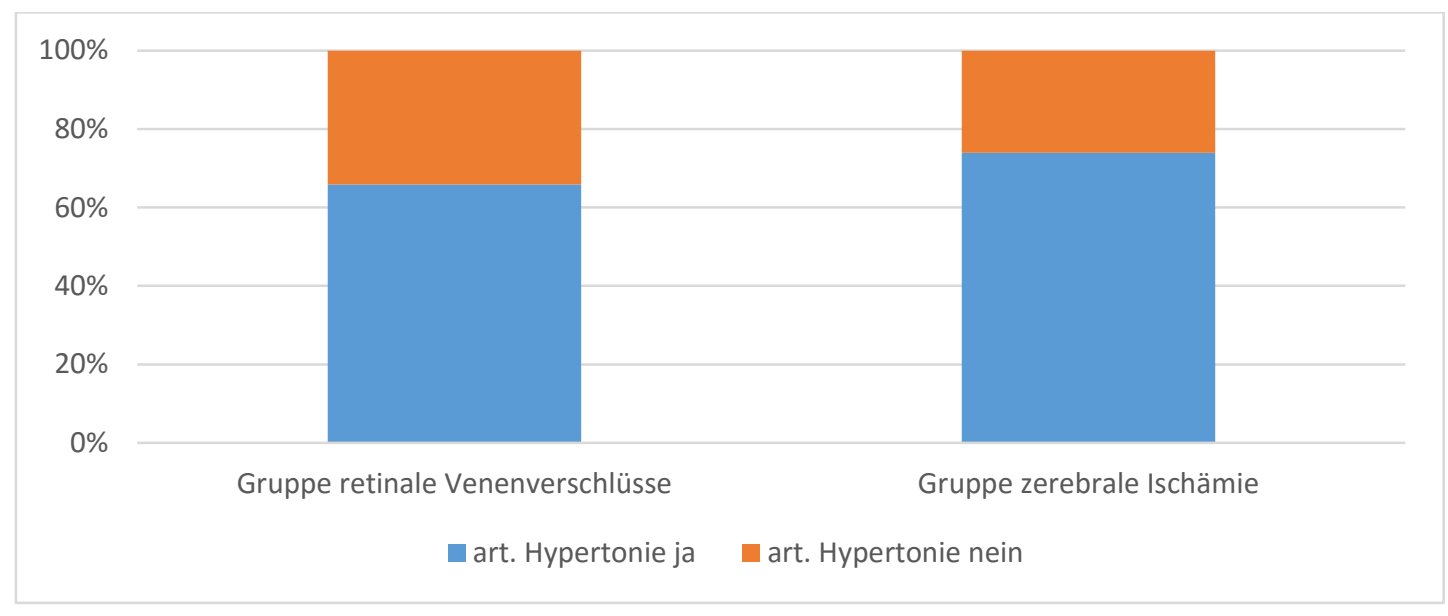

Abbildung 22: Arterielle Hypertonie - Gruppen im Vergleich 
Die nachfolgende Abbildung 23 zeigt die Verteilung der anamnestisch bekannten Hyperlipidämie der beiden Gruppen im Vergleich. Bei den Patienten mit retinalen Venenverschlüssen gaben $28 \%$ und bei den Patienten mit zerebraler Ischämie $34 \%$ an, unter einer Hyperlipidämie zu leiden.

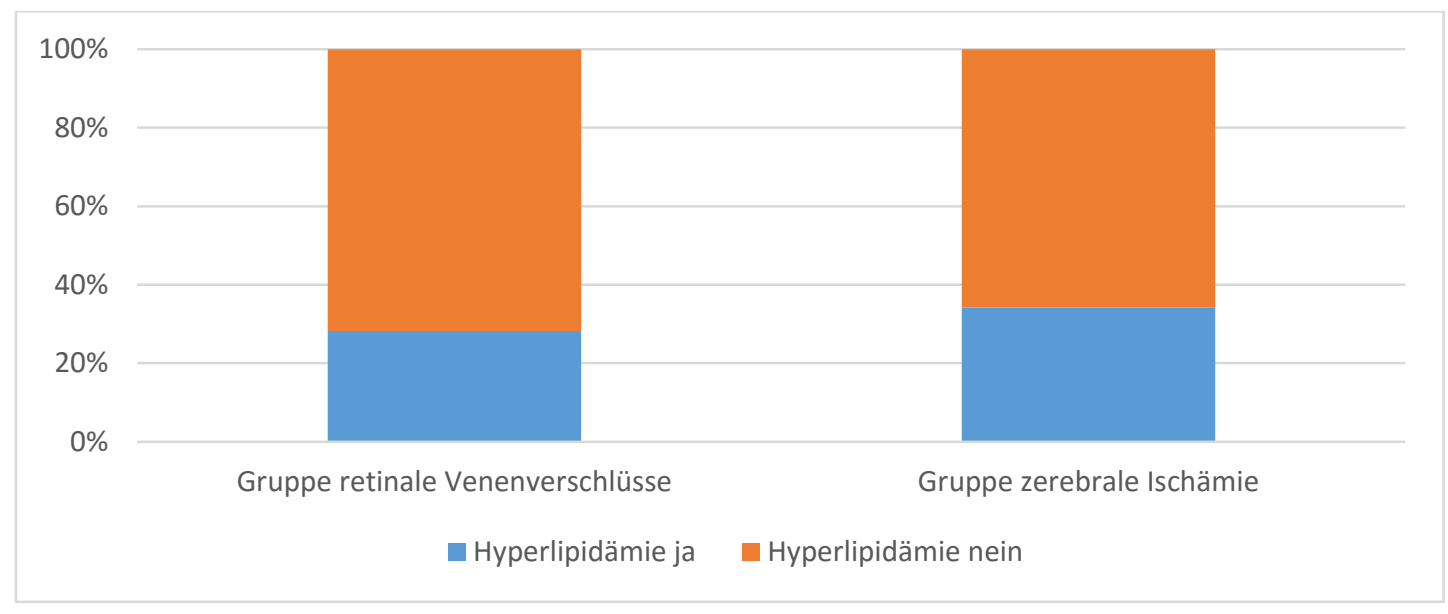

Abbildung 23: Hyperlipidämie - Gruppen im Vergleich

In der Abbildung 24 wird der bei Aufnahme bekannte Anteil der Patienten mit Diabetes mellitus dargestellt. Dies sind $15 \%$ der Patienten mit retinalen Venenverschlüssen und $24 \%$ der Patienten mit zerebraler Ischämie.

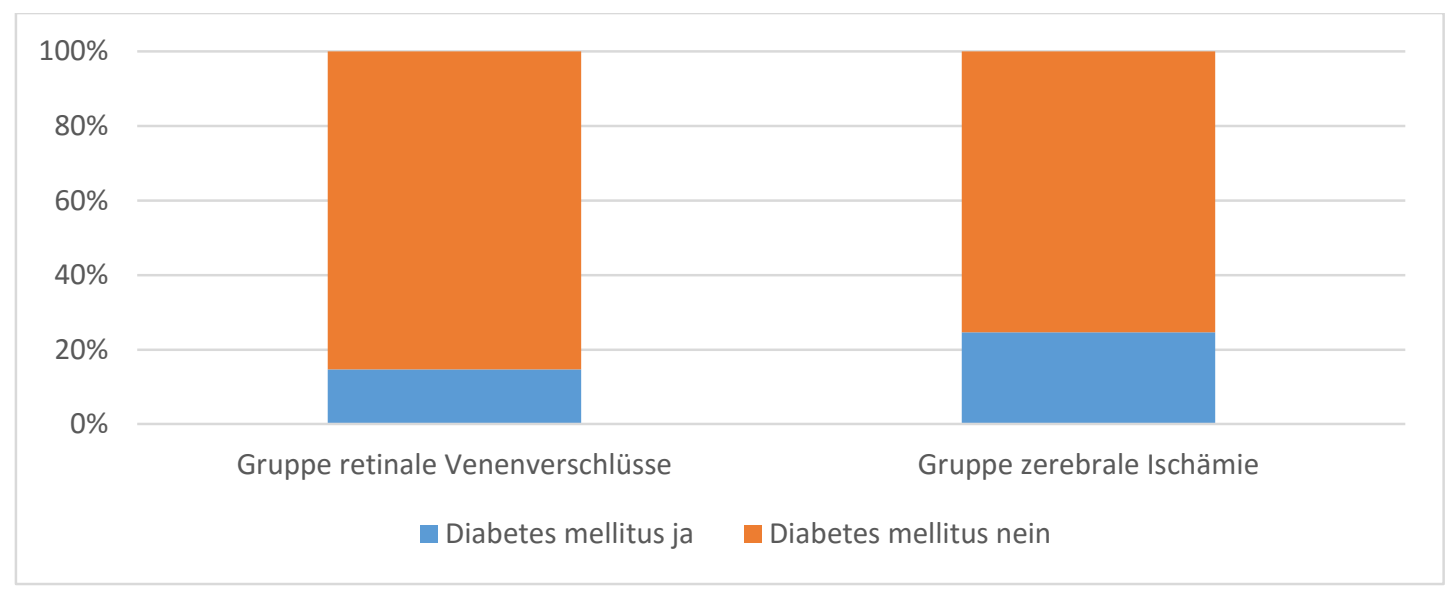

Abbildung 24: Diabetes mellitus - Gruppen im Vergleich 
Die Abbildung 25 zeigt die prozentuale Verteilung der Patienten mit bekanntem und im 7 d-LZ-EKG neu diagnostiziertem Vorhofflimmern. Insgesamt hatten $20 \%$ der Patienten mit venösem Gefäßverschluss im Auge und $26 \%$ der Patienten mit zerebraler Ischämie Vorhofflimmern. Bei $12 \%$ und $16 \%$ der Patienten konnte bereits im Aufnahme-EKG Vorhofflimmern diagnostiziert werden und bei $9 \%$ und $13 \%$ wurde es durch das $7 \mathrm{~d}$-LZ-EKG detektiert.

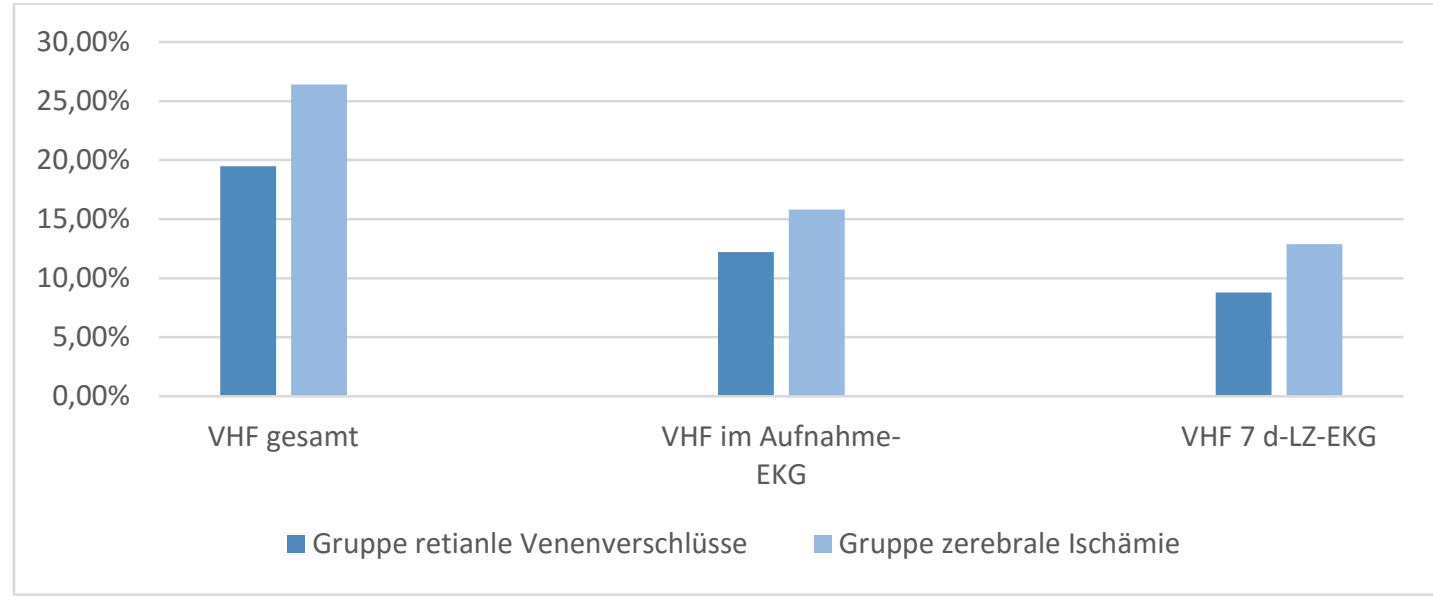

Abbildung 25: Vorhofflimmern - Gruppen im Vergleich 
Die Abbildung 26 zeigt den signifikanten Unterschied der beiden Patientengruppen mit einer $>50 \%$-Karotisstenose $(p=0,03)$. In der Patientengruppe mit retinalen Venenverschlüssen konnte bei $6 \%$ und in der Patientengruppe mit zerebraler Ischämie bei $16 \%$ eine $>50 \%$-Karotisstenose diagnostiziert werden.

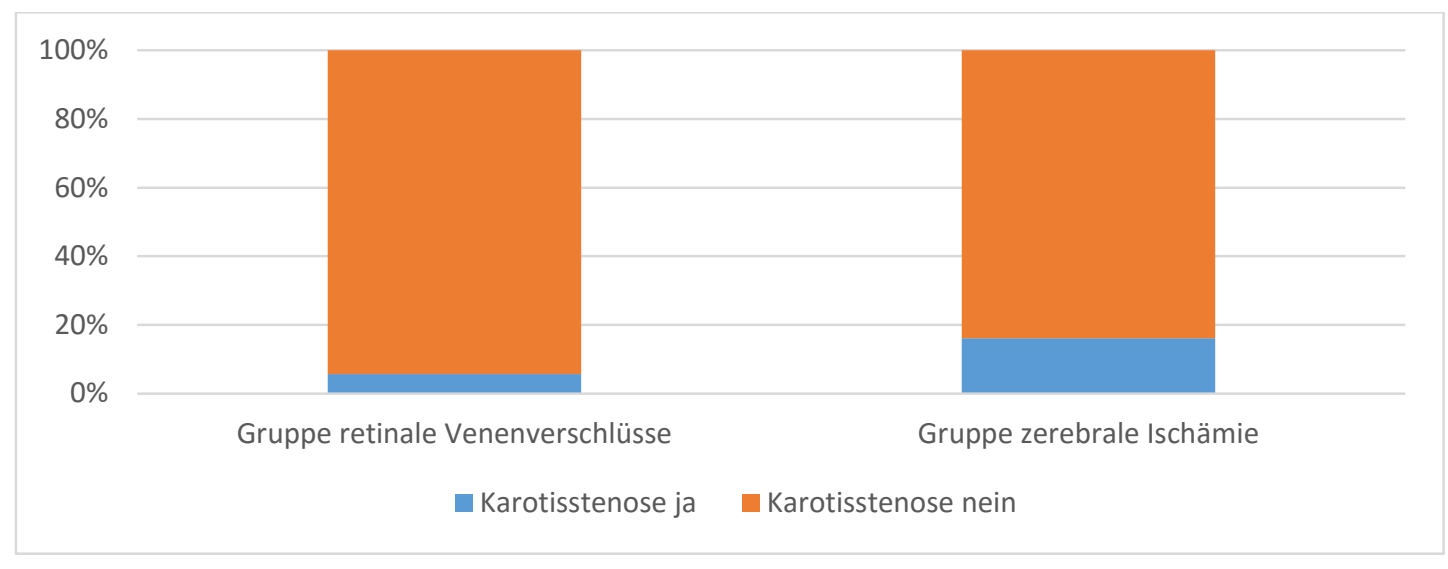

Abbildung 26: Karotisstenose - Gruppen im Vergleich

Die Abbildung 27 zeigt die Verteilung von relevanten Herzklappenveränderungen beider Patientengruppen im Vergleich. Diese wurden in der Gruppe der retinalen Gefäßverschlüsse bei 7 Patienten (21\%) und in der Gruppe der zerebralen Ischämie bei 61 Patienten (25\%) diagnostiziert.

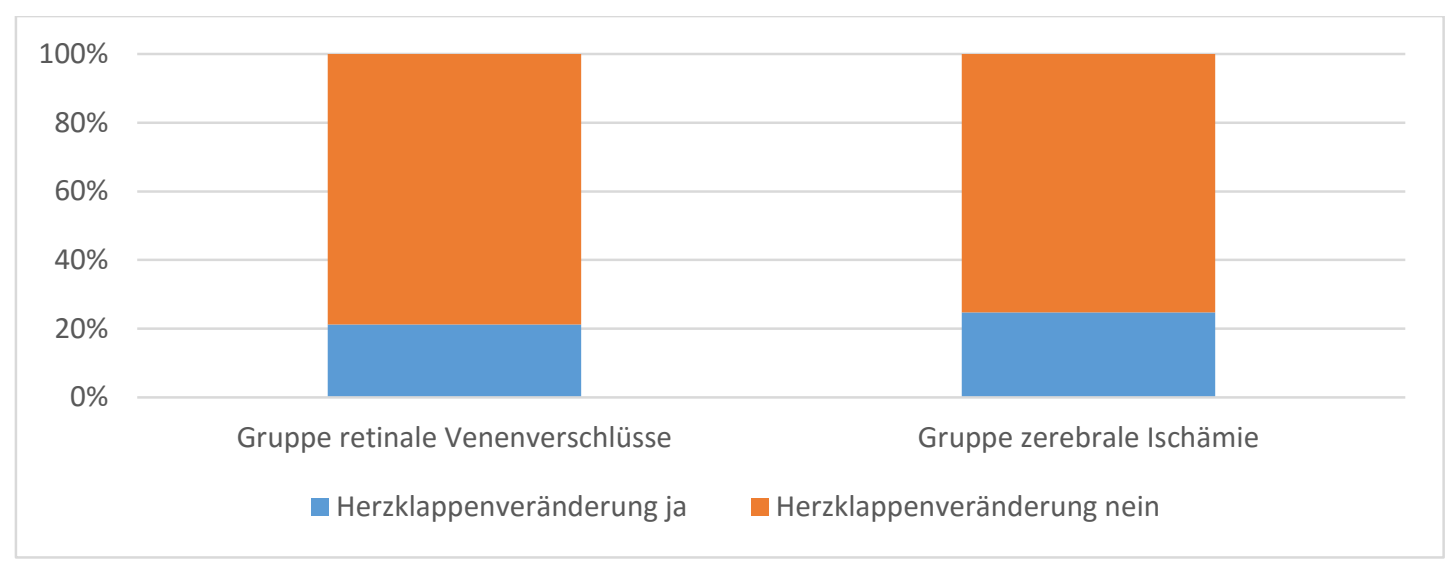

Abbildung 27: Herzklappenveränderung - Gruppen im Vergleich 


\section{Diskussion}

Ziel dieser Arbeit war die prospektive Evaluation kardiovaskulärer Risikofaktoren bei venösen retinalen Gefäßverschlüssen. Es ist davon auszugehen, dass kardiovaskuläre Grunderkrankungen bei der Entstehung von retinalen Venenverschlüssen eine bedeutende Rolle spielen, weshalb es von hoher klinischer Relevanz wäre, kardiovaskuläre Risikofaktoren frühzeitig zu erkennen und zu behandeln.

\subsection{Patientenkollektiv}

Von den 41 Venenverschlüssen im Auge erlitten 26 Patienten einen ZVV, 11 Patienten einen VAV und vier Patienten einen HZVV. In der Literatur sind beide Augen gleichermaßen betroffen (Klein 2008). Dies zeigte sich auch in der vorliegenden Studie, in der mit 53,7 \% das rechte und mit 46,3\% das linke Auge betroffen war. Ein VAV tritt normalerweise häufiger auf als ein ZVV (David et al. 1988). Je nach Lokalisation und Ausdehnung des Ödems oder der Blutung kann ein VAV ohne Symptome bleiben. In unserer Studie wurden nur Patienten mit einem symptomatischen VAV erfasst. Daher ist davon auszugehen, dass Patienten mit einem VAV in unserer Studie unterrepräsentiert sind.

Das Patientenkollektiv aus 41 retinalen Venenverschlüssen weist eine Geschlechterverteilung von 41,5\% weiblichen und 58,5\% männlichen Patienten auf. Männer erleiden häufiger einen VAV als Frauen (Hayreh 1994; Dithmar et al. 2003). Das durchschnittliche Erkrankungsalter liegt zwischen 60 und 70 Jahren, und das Risiko, einen VAV zu erleiden, steigt mit zunehmendem Alter (Dithmar et al. 2003; Rogers et al. 2010a). In unserer 
Studie lag das Alter im Mittel bei 65 Jahren, 24 Patienten (58,5 \%) waren älter als 65 Jahre und 30 Patienten (73,2 \%) älter als 60 Jahre. Es handelt sich, bezogen auf das Alter, um ein repräsentatives Kollektiv.

\subsection{Evaluation kardiovaskulärer Risikofaktoren}

Der arterielle Hypertonus gilt als ein wesentlicher Risikofaktor für kardio- und zerebrovaskuläre Erkrankungen (Trenkwalder 2000). Auch in der vorliegenden Studie ist die arterielle Hypertonie als Hauptrisikofaktor anzusehen. Insgesamt hatten 75,6 \% der Patienten einen arteriellen Hypertonus. Dies deckt sich mit der retrospektiven Fallanalyse aus 2010, dort litten 78,6 \% der Patienten an einem arteriellen Hypertonus (Klatt et al. 2010). Lang beschrieb das Vorkommen zwischen 34-75 \% (Lang und Spraul 1997), Feltgen zwischen 3270 \% (Feltgen 2008). In einer Metaanalyse von O'Mahoney mit 21 Studien im Zeitraum von 1985 bis 2007 wurde bei Patienten mit einer arteriellen Hypertonie ein 3,5-fach erhöhtes Risiko für einen retinalen Venenverschluss beobachtet (O’Mahoney 2008). Bei 63,6 \% der Patienten konnte ein arterieller Hypertonus nachgewiesen werden (O'Mahoney 2008), in der Beaver Dam Eye Study (BDES) und Blue Mountain Eye Study (BMES) sogar bei 89,2 \% und $89,7 \%$ (Cugati et al. 2007).

Die Hyperlipidämie begünstigt ebenso die Entstehung der Arteriosklerose und gilt als ein wesentlicher systemischer Risikofaktor bei retinalen Venenverschlüssen. Patienten mit einer Hyperlipidämie weisen ein 2,5-fach erhöhtes Risiko für ein venöses Verschlussereignis auf (O’Mahoney 2008). In der vorliegenden Studie ist die Hyperlipidämie als zweithäufigster Risikofaktor anzusehen. Insgesamt hatten 35,9 \% der Patienten pathologisch erhöhte Werte des Cholesterins oder der Triglyceride. Dies deckt sich auch mit der 
Metaanalyse von O'Mahoney. Dort konnte bei 35,1 \% der Patienten mit venösem Gefäßverschluss im Auge eine Hyperlipidämie diagnostiziert werden (O’Mahoney 2008).

Strukturelle Herzklappenveränderungen sind häufig Ursache von Emboli des Herzens und spielen als Risikofaktor bei arteriellen Gefäßverschlüssen eine wichtige Rolle (Mirshahi et al. 2008). In der vorliegenden Studie litten 21,2\% der Patienten an relevanten Herzklappenveränderungen. Da Patienten mit venösem Gefäßverschluss ein zweifach erhöhtes Schlaganfallrisiko haben (Werther et al. 2011), ist die transthorakale Echokardiographie bei Patienten mit retinalem Venenverschluss sinnvoll.

Ein weiterer bedeutender Risikofaktor kardiovaskulärer Ereignisse ist das paroxysmale Vorhofflimmern. In einer Arbeit von 2007 konnte ein signifikanter Zusammenhang zwischen dem Auftreten von Vorhofflimmern und retinalen Venenverschlüssen festgestellt werden, wo 18 von 140 (12,9\%) Patienten mit retinalem Venenverschluss und 8 von 142 (5,6 \%) Patienten der Kontrollgruppe Vorhofflimmern hatten (Koizumi et al. 2007). In einer anderen Arbeit von 2009 wurde die Entwicklung eines Schlaganfalls nach retinalem Venenverschluss untersucht (Ho et al. 2009b). Im Alter zwischen 60-69 Jahren weisen Verschlusspatienten ein 2,34-fach erhöhtes Risiko auf, einen Schlaganfall zu bekommen (Ho et al. 2009b). In dieser Altersklasse hatten jedoch nur 3 von 113 (2,7 \%) Verschlusspatienten und keiner der 678 Kontrollpatienten Vorhofflimmern (Ho et al. 2009a). Auch nach fünf Jahren lagen die Zahlen bei 4,4 \% und 0,35 \% nur geringfügig höher (Ho et al. 2009a). Eine weitere Arbeit aus dem Jahr 2013 zeigt wiederum ein erhöhtes Schlaganfallrisiko bei Patienten mit retinalem Venenverschluss und Vorhofflimmern (Christiansen et al. 2013). Der retinale Verschluss wurde 
hierbei sogar als Vorbote eines thromboembolischen Ereignisses gewertet (Christiansen et al. 2013).

Eine Göttinger Studie an Schlaganfallpatienten zeigte, dass durch eine verlängerte elektrokardiographische Aufzeichnungsdauer von sieben Tagen signifikant mehr Patienten mit paroxysmalem Vorhofflimmern identifiziert werden können (Stahrenberg et al. 2010). In der vorliegenden Studie litten insgesamt $8(19,5 \%)$ an Herzrhythmusstörungen, bei Studieneinschluss waren es 5 Patienten (12,2 \%). Durch das Holter-Monitoring über sieben Tage wurde bei 3 (8,8 \%) weiteren Patienten paroxysmales Vorhofflimmern neu diagnostiziert. Betrachtet man alle Patienten mit Herzrhythmusstörungen, identifizierte die verlängerte elektrokardiographische Aufzeichnungsdauer bei mehr als einem Drittel paroxysmales Vorhofflimmern und ist somit bei Patienten mit retinalen Venenverschlüssen von großer klinischer und diagnostischer Bedeutung.

Der Diabetes mellitus ist ein weiterer wichtiger kardiovaskulärer Risikofaktor und führt langfristig sowohl zur Mikro- als auch zur Makroangiopathie. In der vorliegenden Studie hatten 19,5\% der Patienten einen Diabetes mellitus. Auch in O'Mahoney Metaanalyse litten 14,6 \% der Patienten mit einem venösen Gefäßverschluss im Auge an einem Diabetes mellitus (O'Mahoney 2008). In der BDES wurden mit 18,4 \% ähnliche Werte wie in unserem Kollektiv festgestellt wohingegen in der BMES die Zahl der an Diabetes erkrankten Patienten mit 8,6 \% deutlich niedriger lag (Cugati et al. 2007). Auch in einer Arbeit von 2010 hatten nur 7 \% der Patienten einen Diabetes mellitus (Klatt et al. 2010). Ein Grund hierfür könnte sein, dass die Patienten mit einem durchschnittlichen BMI von $25,5 \mathrm{~kg} / \mathrm{m}^{2}$ und $25,8 \mathrm{~kg} / \mathrm{m}^{2}$ deutlich schlanker und im Durchschnitt fast normalgewichtig waren. Der Diabetes mellitus ist nicht nur ein Risikofaktor für retinale Gefäßverschlüsse, sondern die diabetische Retinopathie als Komplikation auch die häufigste Ursache für die Erblindung 
im Alter von 20-74 Jahren (Harris 1998). Um Komplikationen und Spätschäden $\mathrm{zu}$ vermeiden, ist es von klinischer Bedeutung die Blutglukose- und $\mathrm{HbA} 1 \mathrm{c}-$ Werte von Patienten mit retinalen Venenverschlüssen zu untersuchen und bei Bedarf zu behandeln.

Der chronische Nikotinabusus ist ein kardiovaskulärer Risikofaktor von großer Bedeutung, denn alleine der Verzicht von Nikotin reduziert die kardiovaskuläre Mortalität signifikant (Critchley und Capewell 2003). Die toxischen Substanzen schädigen das Endothel und begünstigen die Entstehung von Arteriosklerose (Cacciola et al. 2007). Eine Raucheranamnese hatten in der vorliegenden Studie 21 von 39 Patienten $(53,8$ \%). Davon waren ca. ein Viertel Frauen und drei Viertel Männer. Unter den 21 Patienten gab es 9 (23,1 \%) aktive Raucher. In der BDES waren es mit $18,4 \%$ etwas weniger und in der BMES mit 5,8 \% deutlich weniger Patienten (Cugati et al. 2007). In einer 2013 veröffentlichten Risikoberechnung zeigte sich, dass unter den Patienten mit einem retinalen Venenverschluss, männliche Raucher das höchste Risiko hatten, innerhalb der nächsten zehn Jahre ein kardiovaskuläres Ereignis zu erleiden (Khan et al. 2013). Die Patienten sind auf mögliche gesundheitliche Folgen des Nikotinkonsums hinzuweisen, und es sollte innen dringend zu einem Nikotinstopp geraten werden.

Der Body-Mass-Index (BMI) lag im vorliegenden Patientenkollektiv wie auch in der BDES (BMI 29,2 kg/m²) (Cugati et al. 2007) im Mittel bei $29 \mathrm{~kg} / \mathrm{m}^{2}$ und somit oberhalb des Normalgewichts. Nach der WHO ist ein Wert zwischen 25$29,99 \mathrm{~kg} / \mathrm{m}^{2}$ als Übergewicht einzustufen, ein Wert $\geq 30 \mathrm{~kg} / \mathrm{m}^{2}$ als Adipositas (World Health Organization 2014). In anderen Populationen war der BMI an der Grenze zum Normwert wie beispielsweise in der BMES, dort lag der BMI im Mittel bei $25,5 \mathrm{~kg} / \mathrm{m}^{2}$ (Cugati et al. 2007) und bei der retrospektiven 
Fallanalyse von Klatt lag der BMI im Mittel bei $25,8 \mathrm{~kg} / \mathrm{m}^{2}$ (Klatt et al. 2010). Die unterschiedlichen Werte lassen sich durch die regional unterschiedlichen Populationen der Patientenkollektive erklären. Übergewicht und Adipositas stellen einen bedeutenden Risikofaktor in der Entstehung von retinalen Venenverschlüssen dar.

Welche Rolle die Karotisstenose als Ursache für einen retinalen Venenverschluss einnimmt, ist noch nicht endgültig geklärt. In einer Arbeit von 1984 konnten bei $17 \%$ der Patienten mit einem ZVV eine $\geq 50 \%$ Karotisstenose festgestellt werden (Brown et al. 1984). In einer Arbeit von 2002 wurden bei $6 \%$ der Patienten eine $\geq 70 \%$-Karotisstenose und bei $18 \%$ eine $<70$ \%-Karotisstenose diagnostiziert (Sayag et al. 2002). Auch Wong et al. fanden einen Zusammenhang zwischen dem Auftreten von retinalen Venenverschlüssen und einer Karotisstenose (Wong et al. 2005). Möglicherweise muss hierfür zwischen der nicht-ischämischen und ischämischen Form des Zentralvenenverschlusses unterschieden werden. Karotisstenosen scheinen bei Patienten mit einem ischämischen ZVV eine größere Rolle als bei Patienten mit einem nicht-ischämischen ZVV zu spielen (Hansen 2012). Insgesamt gibt es jedoch keinen Unterschied im Vorkommen von flussrelevanten Karotisstenosen zwischen den Patienten mit retinalem Venenverschluss und den Kontrollpatienten (Hansen 2012). In Arbeiten von 1996 und 2005 wurden ebenfalls vergleichbare Ergebnisse zwischen der Kontrollgruppe mit der Patientengruppe der retinalen Venenverschlüsse gefunden (Rauh et al. 1996; Marin-Sanabria et al. 2005). In der vorliegenden Studie hatten 5,7 \% der Patienten eine $\geq 50 \%$-Karotisstenose. Diese Daten decken sich mit einer retrospektiven Fallanalyse von Klatt et al., in der ein Patient (4,8 \%) im AC-Doppler eine Stenosierung aufwies (Klatt et al. 2010). Somit liegt die Prävalenz einer Karotisstenose in dem Bereich der Normalbevölkerung (<70 Jahre bei 4,8\% $\curvearrowright$ und 2,2 \% $q$ und bei $>70$ Jahre 
bei $12,5 \%$ त und $6,9 \%$ \%) (de Weerd et al. 2009) und scheint kein wesentlicher Risikofaktor für retinale Venenverschlüsse zu sein. In einer Studie von 2011 konnte jedoch bei Patienten mit retinalem Venenverschluss gegenüber der Kontrollgruppe ein zweifach erhöhtes Apoplexrisiko nachgewiesen werden (Werther et al. 2011). Diese Ergebnisse lassen wiederum eine Untersuchung der A. carotis prinzipiell sinnvoll erscheinen, nicht um eine Behandlung des Venenverschlusses zu erreichen, sondern vielmehr um eine zuverlässigere Einschätzung des kardiovaskulären Risikos des individuellen Patienten zu gewinnen.

Einen Apoplex in der Vorgeschichte hatten in der vorliegenden Studie bei Studieneinschluss 2,4 \% der Patienten. In der Literatur findet man unterschiedliche Ergebnisse. Auch in der BDES waren es nur 2,6 \% (Cugati et al. 2007), in einer retrospektiven Fallanalyse hingegen $9,5 \%$ der Patienten (Klatt et al. 2010) und in der BMES 15,8 \% (Cugati et al. 2007). Cugati et al. fanden im männlichen Patientenkollektiv in ihrer Arbeit zudem eine nichtsignifikante, zweifach erhöhte zerebrovaskuläre Mortalität gegenüber der Kontrollgruppe (Cugati et al. 2007). Dies bestätigen weitere Arbeiten. Sie zeigten geschlechterunspezifisch ein zweifach erhöhtes Risiko, nach einem retinalen Venenverschluss auch einen Schlaganfall zu erleiden (Ho et al. 2009b; Werther et al. 2011). In einer Arbeit von 2012 zeigten die Nachuntersuchungen nach acht Jahren, dass 22,2 \% der Patienten mit retinalem Venenverschluss gegenüber 7,6 \% der Patienten der Kontrollgruppe ein kardiovaskuläres oder zerebrovaskuläres Ereignis erlitten hatten (Di Capua et al. 2012).

In der vorliegenden Studie wurden nur die Ereignisse zum Zeitpunkt des Studieneinschlusses betrachtet. Über möglichere spätere Vorfälle gibt es keine bzw. nur unzureichende Angaben. 
Die Koronare Herzkrankheit (KHK) ist die Ausbildung von Arteriosklerose an den Herzkranzgefäßen (Elsner 2006) und neben der arteriellen Hypertonie, der Hyperlipidämie und dem Diabetes mellitus mit 22-50 \% eine der häufigsten kardiovaskulären Risikofaktoren bei Patienten mit venösem Gefäßverschluss im Auge (Hansen 2007).

In einer retrospektiven Analyse von Klatt et al. litten nur 11,9\% der Patienten an einer KHK (Klatt et al. 2010). Auch in der vorliegenden Studie waren es mit 4,9\% deutlich weniger Patienten die anamnestisch eine KHK angaben. Eine $\mathrm{KHK}$ ist bis zu einem gewissen Erkrankungsgrad asymptomatisch, treten Symptome auf, äußern diese sich in Form von Angina pectoris oder einem Myokardinfarkt. In der vorliegenden Studie hatten 7,3 \% der Patienten schon mal einen Myokardinfarkt gehabt. Es lässt sich somit vermuten, dass in dem vorliegenden Kollektiv mehr Patienten von einer KHK betroffen sind. Eine genaue Einteilung der KHK lässt sich nur mit einer Angiographie klären, diese Untersuchung ist nicht Teil der Routineuntersuchung und wurde nicht in das diagnostische Spektrum der Studie aufgenommen.

\subsection{Vergleich einzelner Prävalenzen von Risikofaktoren bei Patienten mit anderen zerebralen Ischämien}

In einem weiteren Schritt wurden einzelne Prävalenzen der Risikofaktoren von dem Patientenkollektiv der retinalen Venenverschlüsse (FIND-AF-EYEStudie) mit einem Patientenkollektiv anderer zerebraler Ischämien (FIND-AFStudie) verglichen. Dabei wurden die signifikanten Unterschiede in den Vordergrund gestellt.

Die retinalen Blutgefäße teilen ähnliche anatomische, physiologische und embryologische Eigenschaften wie die zerebralen Gefäße (Hughes et al. 2000; Patton et al. 2005). Es wird vermutet, dass Gefäßwandveränderungen 
der Netzhautarterie zum retinalem Venenverschluss führen (Seitz 1962) und somit müssen die gleichen Ursachen in der Entstehung von zerebralen Ischämien diskutiert werden (Patton et al. 2005). Das Vergleichskollektiv ist ein Datensatz der FIND-AF-Studie (ISRCTN 46104198), die im Zeitraum von März 2009 bis Februar 2010 an der Universitätsmedizin Göttingen durchgeführt wurde. Es handelte sich ebenfalls um eine prospektive Beobachtungsstudie, in die Patienten mit einem akuten ischämischen Schlaganfall oder einer transitorisch ischämischen Attacke eingeschlossen wurden. Da die Studie aus dem gleichen Zentrum stammt, ist die Durchführung der vorliegenden Studie eng mit dem Design der FIND-AF-EYEStudie verknüpft. Die Durchführung und Auswertung der kardiologischen (TTE, EKG, 7 d-LZ-EKG) und neurologischen (Duplexsonographie) Untersuchungen erfolgte in den gleichen Abteilungen.

Die Rekrutierung der Patienten fand in beiden Studien während eines Jahres statt. In die FIND-AF-Studie wurden ungefähr sechsmal so viele Patienten eingeschlossen wie in die vorliegende Studie. Dies lässt sich durch die höhere Inzidenz des Schlaganfalls erklären. Untersuchungen zeigen, dass ca. ein Fünftel aller hospitalisierten Schlaganfallpatienten einen Reinfarkt haben (Heuschmann et al. 2010). Dies erklärt auch, dass signifikant mehr Patienten der FIND-AF-Studie einen Schlaganfall oder eine TIA in ihrer Vorgeschichte angaben. Zudem gibt es asymptomatische Varianten des Venenastverschlusses und nicht jeder Patient mit einem Venenverschluss im Auge wird klinisch vorstellig. Die Geschlechterverteilung in beiden Kollektiven ist fast identisch. In der Altersverteilung gab es einen signifikanten Unterschied. Die Patienten der FIND-AF-Studie waren im Mittel $70 \pm 12$ [MW \pm SD] Jahre alt. Der Schlaganfall ist eine Erkrankung des höheren Alters und eine Auswertung mehrerer europäischer Schlaganfallregister zeigte, dass das mediane Alter bei 73 Jahren liegt (Heuschmann et al. 2009). 
Der diastolische Blutdruck bei Aufnahme zeigte einen signifikanten Unterschied. Die Messwerte der Patienten mit einer zerebralen Ischämie lagen bei $80 \pm 13,9 \mathrm{mmHg}$, die der Patienten mit einem retinalen Venenverschluss bei $86 \pm 15,9 \mathrm{mmHg}(p=0,03)$. Beide Messwerte liegen aber im Normbereich. Außerdem konnte ein signifikanter Unterschied bei dem Risikofaktor Rauchen festgestellt werden. Das Ergebnis hängt allerdings von dessen Definition ab. Betrachtet man Raucher und ehemalige Raucher zusammen, sind es in der Gruppe der retinalen Venenverschlüsse 53,8 \% der Patienten und in der Gruppe der zerebralen Ischämie 37,1\% der Patienten $(p=0,05)$, vergleicht man hingegen nur die aktiven Raucher zeigt sich zwischen den Gruppen kein Unterschied mehr. In der Gruppe der venösen Gefäßverschlüsse im Auge sind unter den 21 Patienten nur 9 Patienten $(23,1 \%)$ aktive Raucher und in der Gruppe der zerebralen Ischämie von 101 Patienten nur 57 Patienten (21\%) aktive Raucher.

Ein signifikanter Unterschied zeigte sich beim Vergleich der Prävalenzen der Karotisstenose $(p=0,03)$. Die Prävalenz des Kollektivs der retinalen Venenverschlüsse liegt dabei im dem Bereich der Normalbevölkerung. Das Ergebnis zeigt einen Trend, denn aufgrund der hohen Fehlquote der duplexsonographischen Untersuchungen bei den Patienten mit retinalen Venenverschlüssen kann es nur einschränkend betrachtet werden. Bei akuten arteriellen Verschlüssen wie beispielsweise bei einer zerebralen Ischämie, liegt in den meisten Fällen ein thromboembolisches Ereignis zugrunde. Venöse Verschlüsse entstehen eher multifaktoriell, arteriosklerotische Veränderungen der eng benachbarten Zentralarterie können dabei zur Kompression und somit zur Thrombenbildung in der Zentralvene führen (Lang 2007; Seidel et al. 2014). 


\subsection{Limitationen}

Die Rekrutierung von Verschlusspatienten für die FIND-AF-EYE-Studie an der Universitätsmedizin Göttingen über ein Jahr führte zu einer limitierten Fallzahl von 41 Patienten mit retinalem Venenverschluss. Zudem ist durch die fehlende Kontrollgruppe gesunder Patienten die Aussagekraft FIND-AF-EYE-Studie eingeschränkt. Die geringe Fallzahl der Verschlusspatienten liegt zum einen an der niedrigen Prävalenz des Krankheitsbildes des retinalen Venenverschlusses und zum anderen an der Durchführung einer monozentrischen Studie.

\subsection{Konsequenz}

Kardiovaskuläre Grunderkrankungen wie beispielsweise die arterielle Hypertonie, die Hyperlipidämie oder der Diabetes mellitus spielen eine entscheidende Rolle in der Entstehung von retinalen Venenverschlüssen. Zudem weisen diese Patienten eine erhöhte kardiovaskuläre Mortalität im Vergleich zur Normalbevölkerung auf (Lang 2007). Deshalb ist eine interdisziplinäre Ursachenabklärung von großer Bedeutung.

Anhand der Ergebnisse der vorliegenden Studie sind apparative Untersuchungen wie eine 24 h-Blutdruckmessung, eine Echokardiographie und ein 7-Tage-Langzeit-EKG unverzichtbar. Laborchemisch sollten ein Lipidstatus und ein Blutzuckerprofil routinemäßig erhoben werden. Unsere Ergebnisse sprechen gegen die Durchführung weiterer apparativer Untersuchung der A. carotis (Duplexsonographie) als Ursachensuche. Gleiche Empfehlungen wurden auch in Arbeiten von 2008 (Mirshahi et al. 2008) und 
2014 (Seidel et al. 2014) ausgesprochen. Jüngste Ergebnisse zeigen jedoch ein erhöhtes Schlaganfallrisiko bei Patienten mit retinalen Venenverschlüssen (Bertelsen et al. 2012; Christiansen et al. 2013), und es kommen erste Zweifel, ob die bisherige Routinediagnostik ausgeweitet werden sollte (Feltgen und Franko Zeitz 2014). Zur Einschätzung des kardiovaskulären Risikos des Patienten erscheint eine Untersuchung der A. carotis sinnvoll. Das Patientenkollektiv der vorliegenden Studie ist mit 41 Patienten zwar ein repräsentatives Kollektiv, aber durch die hohe Fehlquote der duplexsonographischen und echokardiographischen Untersuchungen müssen weitere Studien mit größeren Fallzahlen folgen.

In der folgenden Tabelle sind die empfohlenen interdisziplinären Untersuchungen im Überblick dargestellt.

\footnotetext{
- 24 h-RR-Messung

- Echokardiographie

- 7 d-LZ-EKG

- Duplexsonographie der A .carotis

- Lipidstatus (laborchemisch)

- Blutzuckerprofil (laborchemisch)
}

Tabelle 10: Empfohlene interdisziplinäre Untersuchungen 


\section{Zusammenfassung}

Retinale Venenverschlüsse sind eine der häufigsten vaskulären Netzhauterkrankungen. Bei der Entstehung und dem Verlauf von venösen Gefäßverschlüssen im Auge spielen kardiovaskuläre Risikofaktoren eine entscheidende Rolle. Patienten mit einem retinalen Venenverschluss weisen vermehrt vaskuläre Risikofaktoren für arterielle Gefäßkrankheiten auf, weswegen es von hoher klinischer Relevanz ist, diese frühzeitig zu erkennen und zu behandeln. Bisher gibt es kein einheitliches Untersuchungsschema zur Ursachenabklärung eines retinalen Venenverschlusses. Ziel ist es, Empfehlungen einer zukünftigen Routinediagnostik für venöse Gefäßverschlüsse im Auge auszusprechen.

In der FIND-AF-EYE-Studie wurde erstmals durch eine umfangreiche Diagnostik an insgesamt 101 Patienten mit retinalen Gefäßverschlüssen eine systematische Abklärung kardiovaskulärer Risikofaktoren kontrolliert durchgeführt. Die Diagnostik umfasste eine duplexsonographische Untersuchung der A. carotis, eine Echokardiographie, eine 24 h-Blutdruckmessung, ein 7 d-LZ-EKG und eine laborchemische Erhebung des Lipidund Glukosestatus.

Bei der Auswertung der 41 Patienten mit venösen Gefäßverschlüssen im Auge konnte wie auch in vergleichbaren Studien gezeigt werden, dass vor allem die arterielle Hypertonie, der Nikotinkonsum, die Hyperlipidämie und der Diabetes mellitus die wichtigsten kardiovaskulären Risikofaktoren darstellen.

Zudem wurde durch die verlängerte elektrokardiographische Aufzeichnungsdauer über sieben Tage mehr als ein Drittel aller Patienten mit Herzrhythmusstörungen identifiziert. 
Bei anderen kardiovaskulären Grunderkrankungen, wie beispielsweise der Karotisstenose, lag die Prävalenz hingegen nur in dem Bereich der gleichaltrigen Allgemeinbevölkerung.

Anhand der Ergebnisse der vorliegenden Studie sind apparative Untersuchungen wie eine 24 h-Blutdruckmessung, eine Echokardiographie und ein 7-Tage-Langzeit-EKG unverzichtbar. Laborchemisch sollten ein Lipidstatus und ein Blutzuckerprofil routinemäßig erhoben werden. Zur Einschätzung des kardiovaskulären Risikos des Patienten erscheint eine Untersuchung der A. carotis sinnvoll.

Um die Morbidität und die Mortalität zu senken bedarf es einer interdisziplinären Ursachenabklärung, welches die enge Zusammenarbeit zwischen Ophthalmologen und Internisten erfordert.

Vergleich von Prävalenzen einzelner Risikofaktoren mit anderen zerebralen Ischämien

Vergleicht man die FIND-AF-EYE-Studie mit der bereits publizierten FIND-AFStudie litten die Patienten der FIND-AF-Studie signifikant häufiger an einer Karotisstenose.

Zusammenfassend lässt sich sagen, dass akuten arteriellen Verschlüssen, wie beispielsweise einer zerebralen Ischämie, in den meisten Fällen thromboembolische Ereignisse zugrunde liegen. Kardiovaskuläre Risikofaktoren spielen aber auch bei venösen Gefäßverschlüssen im Auge eine wichtige Rolle. Hierbei führen sie zu arteriosklerotischen Veränderungen der eng benachbarten Zentralarterie. Durch die Kompression kommt es somit zur Thrombenbildung in der Zentralvene. 


\title{
6 Anhang
}

\subsection{Patienteninformation}

\author{
Zentrum 13 - Augenklinik mit Poliklinik \\ Abteilung Augenheilkunde \\ Direktor. Professor Dr. med. Hans Hoerauf \\ Studienleiter. PD Dr. med. Nicolas Feltgen \\ Robert-Koch-Strasse 40, 37075 Göttingen \\ Tel-0551-39 6778/ Fax 0551-39 6787 \\ E-mail: nicolas.feltgen@med.uni-goettingen.de
}

GÖIINGEN $=\mathbf{U} \mathbf{V}$

\section{RISIKOSTRATIFIZIERUNG BEI PATIENTEN MIT ARTERIELLER ODER VENÖSER THROMBOSE DER AUGENGEFÄßE}

\author{
- Patienteninformation -
}

Sehr geehrte Patientin, sehr geehrter Patient,

wir möchten Sie um die freiwillige Teilnahme an einer wissenschaftlichen Studie bitten.

Sie haben einen Gefäßverschluss am Auge erlitten (Verschluss einer Netzhautvene, einer Netzhautarterie oder einer versorgenden Arterie des Sehnerven). Eine mögliche Ursache dafür sind Herzrhythmusstörungen, die man im Elektrokardiogramm (EKG) nachweisen kann.

Oft treten diese Herzrhythmusstörungen nur anfallsweise (paroxysmal) auf, das heißt, man muss regelmäßig und öfter ein EKG schreiben. Es besteht die Vermutung, dass man eine Herzhythmusstörung besser mit einem kontinuierlichen EKG (Langzeit-EKG) entdecken könnte. Herzrhythmusstörung besser mit einem kontinuierlichen EKG (Langzeit-EKG) entdecken könnte.
Bisher erfolgt die Abklärung der Herzrhythmusstörungen mit einem Langzeit-EKG nicht Bisher erfolgt die Abklärung der Herzhythmusstörungen mit einem Langzeit-EKG nicht ambulanten Betreuung durch lhren Hausarzt. Ob ein Langzeit-EKG wirklich dazu führt, dass Herzrhythmusstörungen besser erkannt werden, ist die Frage, die dieser Studie zugrunde liegt.

Leider gibt es bisher kaum Daten darūber, wie hāufig und wann Patienten mit Gefäßverschluss am Auge auch an Herzrhythmusstörungen leiden. Dieses Wissen würde uns im klinischen Alltag aber helfen, Untersuchungen gezielter einzusetzen. Wir möchten Sie deshalb bitten, uns bei eine wissenschaftlichen Studie zu unterstützen, die wir bei Patienten mit Gefäßverschluss am Auge durchführen möchten. Die Teilnahme an dieser wissenschaftlichen Studie ist freiwillig. Die Entscheidung, ob Sie an dieser wissenschaftlichen Studie teilnehmen oder nicht, hat keinen Einfluss auf Ihre weitere augenärztliche Behandlung. Falls Herzrhythmusstörungen gefunden werden sollten, würde diese Information aber sehr wahrscheinlich lhre internistische Behandlung werden sollten, würde diese Information aber sehr wahrscheinlich Ihre internistische Behandlung
verändern (z.B. Einnahme von gerinnungshemmenden Medikamenten wie Marcumar oder ASS etc).

Ihre Teilnahme an dieser Studie ist freiwillig. Sie werden in diese Studie also nur dann einbezogen, wenn Sie dazu schriftlich lhre Einwilligung erklären. Sofern Sie nicht an der Studie teilnehmen oder später aus ihr ausscheiden möchten, erwachsen lhnen daraus keine Nachteile. 
Bitte lesen Sie die nachstehende Information sorgfältig. Bei Unklarheiten wird Ihr Arzt Sie ausführlich beraten.

Was ist das Ziel der Studie?

Ziel unserer Untersuchung ist die genauere Erforschung der Hāufigkeit (Prävalenz) des anfallsweise auftretenden Vorhofflimmerns bei Patienten mit einem Gefäßverschluss am Auge. Außerdem wollen wir feststellen, ob wir Patienten mit internistischen Risikofaktoren mit Hilfe eines Bluttests besser identifizieren können, da bestimmte Botenstoffe (=Hormone) bei bestimmten Herzerkrankungen vermehrt gebildet werden. Es muss Ihnen also auch Blut abgenommen werden.

\section{Was sind die Voraussetzungen für eine Teilnahme?}

Voraussetzung zur Teilnahme ist, dass bei Ihnen ein Verschluss eines Augengefäßes vorliegt, der nicht älter als 4 Wochen ist. Nach eingehender mündlicher und schriftlicher Aufklärung über den Sinn und den Ablauf der Studie werden wir Sie bitten, schriftlich lhr Einverständnis zur Teilnahme zu bekunden.

Welche Untersuchungen werden durchgeführt?

Zu dem Zeitpunkt der Frage, ob Sie an der Studie teilnehmen möchten oder nicht, ist es noch nicht gesichert, ob bei Ihnen eine Herzhythmusstörung vorliegt. Diese Entscheidung hängt vom ersten EKG ab, welches wir möglichst bald durchführen werden.

Innerhalb des stationāren Aufenthaltes werden wir den Herzrhythmus mit einem 7-TageLangzeit-EKG durchgehend aufzeichnen, sofem im Aufnahme-EKG kein Vorhofflimmern vorliegt.

Falls im Aufnahme-EKG eine Herzrhythmusstörung festgestellt werden sollte, würde kein Langzeit-EKG angeschlossen werden, da der Nachweis der Herzhythmusstörung bereits erbracht ist. In diesem Fall ist die Herzhythmusstörung die wahrscheinlichste Ursache des Gefäßverschlusses am Auge und die weiteren diagnostischen und therapeutischen Maßnahmen würden in diese Richtung zielen.

Zusätzlich zur üblichen Standarddiagnostik nehmen wir Ihnen für diese Studie einmalig ca. 7,5 ml Blut ab. Dabei wird die Blutentnahme zeitlich möglichst mit ohnehin geplanten Blutabnahmen zusammengelegt.

Nach einem und nach 12 Monaten möchten wir Sie noch einmal zu einer ambulanten augenärztlichen Untersuchung zu uns einladen.

Was sind die möglichen Risiken oder Nachteile einer Teilnahme an der Studie?

Die Risiken der Studienteilnahme beschränken sich auf die Risiken der durchgeführten Untersuchungen.

1. Die Risiken des Langzeit-EKGs sind ähnlich den Risiken eines sonst durchgeführten EKGs (z. B. allergische Reaktion auf Elektroden).

2. Das Risiko der Blutabnahme (z. B. Hämatom=,blauer Fleck*) ist das gleiche Risiko wie bei jeder anderen Blutabnahme aus einer Armvene. Es können blaue Flecken im Bereich der Einstichstelle entstehen und es besteht das sehr geringe Risiko einer Iokalen oder allgemeinen Infektion. In extrem seltenen Fällen kann es zu einer Verletzung eines Hautnervs, eventuell sogar mit dauerhaftem Verlauf kommen.

Welchen Vorteil haben Sie durch Ihre Teilnahme an der Studie?

Eigennutzen: Durch die Teilnahme an der Studie gewinnen wir Informationen über Ihren Herzhythmus, der möglicherweise die internistische Behandlung ändert ( $z$.B. bei Herzrhythmusstörungen).

Gruppennutzen: Die Ergebnisse der Untersuchungen könnten in der Zukunft helfen, Patienten mit Gefäßverschluss am Auge zu identifizieren, bei denen man gezielter nach Herzhythmusstörungen suchen muss. 
Fremdnutzen: Durch Ihre Teilnahme helfen Sie mit, Vorhofflimmern zukünftig besser zu diagnostizieren, so dass wir in Zukunft andere Patienten auch mit anderen Erkrankungen möglicherweise früher und besser behandeln können.

Was geschieht mit lhren medizinischen Daten bzw. den entnommenen Blutprobe? Es gilt die ärztliche Schweigepflicht. Jede Information über Sie wird zu jeder Zeit mit der größtmöglichen Vertraulichkeit behandelt. Die medizinischen Daten und Messergebnisse werden in einer zentralen Datenbank gespeichert, die den jeweils geltenden datenschutzrechtlichen Auflagen Rechnung trägt. In keiner wissenschaftlichen Veröffentlichung wird Ihr Name erscheinen. Ihre Daten werden auf keinen Fall bekannt gegeben. Die jeweiligen Blutproben werden eingefroren, um auch für später sich ergebende studienspezifische Fragestellungen verwendet werden zu können, die im Zusammenhang mit der aktuellen Grunderkrankung bzw. ihrer Therapie stehen. Das bedeutet, dass die Blutproben nicht für andere Studien verwendet werden, sondern sich die Analyse ausschließlich auf die verbesserte Risikoanalyse des Gefäßverschlusses am Auge bezieht. Sollten weitere Analysen in Frage kommen, würden wir emeut um Ihre schriftliche Einwilligung bitten. Auf keiner Blutprobe steht ihr Name oder sonstige persönliche Informationen über sie. Alle Blutwerte werden verblindet (d. h. ohne Informationen über Ihr Alter, Geschlecht, Namen etc.) gemessen

Ihre personenbezogenen Studiendaten werden für 10 Jahre gespeichert, spätestens nach 15 Jahren werden die Blutproben fachgerecht vernichtet.

Sie kōnnen jederzeit, auch ohne Angabe von Gründen, Ihre Teilnahme beenden, ohne dass Ihnen dadurch irgendwelche Nachteile bei Ihrer medizinischen Behandlung entstehen.

Im Falle einer Rücknahme der Einwilligung werden auf ihren Wunsch hin die bereits erhobenen personenbezogenen Daten gelöscht und die Blutproben fachgerecht vernichtet. 


\title{
6.2 Einverständniserklärung
}

\author{
Zentrum 13 - Augenklinik mit Poliklinik \\ Abteilung Augenheilkunde \\ Direktor. Professor Dr. med. Hans Hoerauf
Studienleiter. PD Dr. med. Nicolas Feltgen \\ Robert-Koch-Strasse 40, 37075 Göttingen \\ Robert-Koch-Strasse 40, 37075 Götting
Tel.: 0551-39 6776/ Fax: 0551-39 6787 \\ E-mail: nicolas.feltgen@med.uni-goettingen.de
}

$\begin{aligned} \text { UNIVERSITATSMEDIZIN } & =1 \mathrm{MO} \\ \text { GOITINGEN } & =\mathbf{M}\end{aligned}$

\section{RISIKOSTRATIFIZIERUNG BEI PATIENTEN MIT ARTERIELLER ODER VENÖSER THROMBOSE DER AUGENGEFÄßE \\ - Einverständniserklärung -}

Ich, meinem Arzt vollständig ūber Wesen, Bedeutung und Tragweite der klinischen Prüfung mit dem o.g. Titel aufgeklärt. Ich habe den Aufklärungstext gelesen und verstanden. Ich hatte die Möglichkeit, Fragen zu stellen, habe die Antworten verstanden und akzeptiere sie. Mein Arzt hat mich über die mit der Teilnahme an der Studie verbundenen Risiken und den möglichen Nutzen informiert.

Ich hatte ausreichend Zeit, mich zur Teilnahme an dieser Studie zu entscheiden und weiß, dass die Teilnahme an dieser klinischen Prüfung freiwillig ist. Ich weiß, dass ich jederzeit und ohne Angaben von Gründen diese Zustimmung widerrufen kann, ohne dass sich dieser Entschluss nachteilig auf die spātere Behandlung durch meinen Arzt auswirken wird. Die von mir gewonnenen Daten und Proben werden im Falle eines Studienrücktritts vernichtet.

Bei dieser wissenschaftlichen Studie werden persönliche Daten und medizinische Befunde über mich erhoben, gespeichert und verarbeitet. Die Speicherung, Auswertung und Weitergabe dieser studienbezogenen Daten erfolgt nach gesetzlichen Bestimmungen und setzt vor Teilnahme an der Studie folgende freiwillige Einwilligung voraus:

1. Ich erkläre mich damit einverstanden, dass im Rahmen dieser Studie erhobene Daten/ Krankheitsdaten auf Fragebögen und elektronischen Datenträgern aufgezeichnet und ohne Namensnennung (verschlüsselt) verarbeitet werden.

2. Ich bin darüber aufgeklärt worden, dass ich jederzeit die Teilnahme an der wissenschaftlichen Prüfung beenden kann. Beim Widerruf meiner Einwilligung an der Studie teilzunehmen, werden meine bis dahin gespeicherten personenbezogenen Daten gelöscht und die Blutproben auf Anfrage fachgerecht entsorgt.

Mir ist bekannt, dass alle Informationen über mich zu jeder Zeit mit der größtmöglichen Vertraulichkeit behandelt werden. Die medizinischen Daten und Messergebnisse werden in einer zentralen Datenbank gespeichert, die den jeweils geltenden datenschutzrechtlichen Auflagen Rechnung trägt. In keiner wissenschaftlichen Veröffentlichung wird mein Name erscheinen. Die personenbezogenen Studiendaten werden für 10 Jahre gespeichert, die Blutproben werden für längstens 15 Jahre aufbewahrt. Danach werden sowohl die Daten gelöscht, als auch die Blutproben fachgerecht entsorgt. 
Ich bin damit einverstanden, dass meine Proben zur weiteren Forschung in Bezug auf die Grunderkrankung aufbewahrt werden.

Ja $\square \quad$ Nein $\square$

Meine Einwilligung bezieht sich auch auf Forschung neu beschriebener Risikofaktoren von HerzKreislauferkrankungen, die zum Zeitpunkt der Einwilligung noch nicht routinemäßig bestimmt werden.

Ja $\square \quad$ Nein $\square$

Ort und Datum Unterschrift des Patienten

Ort und Datum Unterschrift des aufiklärenden Arztes 


\subsection{Abkürzungsverzeichnis}

$\begin{array}{ll}\text { A. } & \text { Arteria } \\ \text { Aa. } & \text { Arteriae } \\ \text { Abb. } & \text { Abbildung } \\ \text { AC } & \text { Arteria Carotis } \\ \text { ACE } & \text { Angiotensin-Converting-Enzym } \\ \text { AF } & \text { atrial firbrillation } \\ \text { AION } & \text { anteriore ischämische Optikusneuropathie } \\ \text { AK } & \text { Antikörper } \\ \text { APC } & \text { aktiviertes Protein C } \\ \text { aPTT } & \text { aktivierte partielle Thromboplastinzeit } \\ \text { art. } & \text { arteriell } \\ \text { BDES } & \text { Beaver Dam Eye Study } \\ \text { BMES } & \text { Blue Mountain Eye Study } \\ \text { BMI } & \text { Body-Mass-Index } \\ \text { BSG } & \text { Blutsenkungsgeschwindigkeit } \\ \text { cm } & \text { Zentimeter } \\ \text { CRP } & \text { C-reaktives Protein } \\ \text { CT } & \text { Computertomographie } \\ \text { d } & \text { Tag(e) } \\ \text { dl } & \text { Deziliter } \\ \text { EDTA } & \text { Ethylendiamintetraacetat } \\ \text { ehem. } & \text { ehemalig } \\ \text { EKG } & \text { Elektrokardiogramm } \\ \text { FRS } & \text { Framingham-Risiko-Score } \\ \text { h } & \text { Stunde(n) } \\ \text { HbA1c } & \text { Glykoprotein } \\ \text { HDL } & \text { high density lipoprotein } \\ & \end{array}$




$\begin{array}{ll}\text { HZVV } & \text { Hemi-Zentralvenenverschluss } \\ \text { ICR } & \text { Interkostalraum } \\ \text { INR } & \text { International Normalized Ratio } \\ \text { ISRCTN } & \text { International Standard Randomised Controlled Trail Number } \\ \text { kg } & \text { Kilogramm } \\ \text { KHK } & \text { koronare Herzkrankheit } \\ \text { LDL } & \text { low density lipoprotein } \\ \text { logMAR } & \text { logarithm of Minimum Angle of Resolution } \\ \text { LZ } & \text { Langzeit } \\ \text { m² } & \text { Quadratmeter } \\ \text { MCV } & \text { mean corpuscular volume } \\ \text { mg } & \text { Milligramm } \\ \text { min } & \text { Minuten } \\ \text { Min } & \text { Minimum } \\ \text { Max } & \text { Maximum } \\ \text { ml } & \text { Milliliter } \\ \text { mmHg } & \text { Millimeter Quecksilbersäule } \\ \text { MW } & \text { Mittelwert } \\ \text { OCT } & \text { optische Kohärenztomographie } \\ \text { OP } & \text { Operation } \\ \text { pAVK } & \text { periphere arterielle Verschlusskrankheit } \\ \text { PD } & \text { Papillendurchmesser } \\ \text { PFO } & \text { persistierendes Foramen ovale } \\ \text { RR } & \text { Blutdruckmessung nach Riva-Rocci } \\ \text { s. } & \text { siehe } \\ \text { SD } & \text { standard deviation } \\ \text { TIA } & \text { transistorische ischämische Attacke } \\ \text { TSH } & \text { Thyreoidea-stimulierndes Hormon } \\ \text { TTE } & \text { transthorakale Echokardiographie } \\ & \\ & \end{array}$




$\begin{array}{ll}\text { WHO } & \text { World Health Organization } \\ \text { V. } & \text { Vena } \\ \text { VAV } & \text { Venenastverschluss } \\ \text { VEGF } & \text { vascular endothelial growth factor } \\ \text { VHF } & \text { Vorhofflimmern } \\ \text { y } & \text { Jahr(e) } \\ \text { ZVV } & \text { Zentralvenenverschluss } \\ { }^{\circ} \mathrm{C} & \text { Grad Celsius }\end{array}$




\subsection{Abbildungsverzeichnis}

Abbildung 1: Einteilung retinale Venenverschlüsse............................... 2

Abbildung 2: Zentralvenenverschlüsse ............................................ 4

Abbildung 3: HZVV in der oberen Hälfte .......................................... 5

Abbildung 4: VAV der V. temporalis inferior ......................................... 5

Abbildung 5: Histologischer Befund eines VAV ..................................... 6

Abbildung 6: Pathogenese des Venenastverschlusses............................ 8

Abbildung 7: Zeitlicher Studienablauf.................................................. 15

Abbildung 8: Elektrodenplatzierung $7 \mathrm{~d}$-LZ-EKG ................................ 21

Abbildung 9: Verteilung der Gefäßverschlüsse .................................. 24

Abbildung 10: Verteilung der venösen Gefäßverschlüsse ....................... 24

Abbildung 11: Verteilung der betroffenen Augen ................................. 25

Abbildung 12: Bekannte und neu diagnostizierte Risikofaktoren ................ 30

Abbildung 13: Geschlechterverteilung - Gruppen im Vergleich.................. 31

Abbildung 14: Boxplot Alter - Gruppen im Vergleich .............................. 32

Abbildung 15: Boxplot BMI - Gruppen im Vergleich ............................... 33

Abbildung 16: Boxplot systolischer Blutdruck - Gruppen im Vergleich ......... 34

Abbildung 17: Boxplot diastolischer Blutdruck - Gruppen im Vergleich........ 35

Abbildung 18: Boxplot Herzfrequenz - Gruppen im Vergleich .................... 36

Abbildung 19: Schlaganfall - Gruppen im Vergleich ............................. 37

Abbildung 20: TIA - Gruppen im Vergleich ..................................... 37

Abbildung 21: Raucheranamnese - Gruppen im Vergleich ....................... 38

Abbildung 22: Arterielle Hypertonie - Gruppen im Vergleich ...................... 39

Abbildung 23: Hyperlipidämie - Gruppen im Vergleich........................... 40

Abbildung 24: Diabetes mellitus - Gruppen im Vergleich ........................ 40

Abbildung 25: Vorhofflimmern - Gruppen im Vergleich ........................... 41

Abbildung 26: Karotisstenose - Gruppen im Vergleich........................... 42

Abbildung 27: Herzklappenveränderung - Gruppen im Vergleich .............. 42 


\subsection{Tabellenverzeichnis}

Tabelle 1: Risikofaktoren für retinale Venenverschlüsse ........................ 10

Tabelle 2: Gruppenzuordnung..................................................... 16

Tabelle 3: Anamnestisch systematisch abgefragte Vorerkrankungen .......... 17

Tabelle 4: Medikamentenanamnese ................................................ 18

Tabelle 5: Ophthalmologische Vorerkrankungen.................................. 18

Tabelle 6: Klinische Untersuchungsparameter .................................. 18

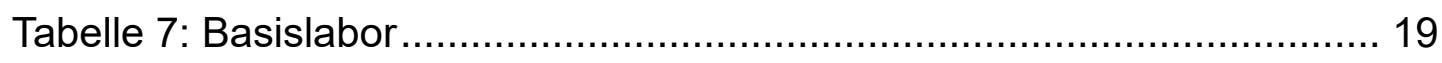

Tabelle 8: Nüchternlabordiagnostik ............................................. 19

Tabelle 9: Klinische Charakteristika - Gruppen im Vergleich ..................... 27

Tabelle 10: Empfohlene interdisziplinäre Untersuchungen ..................... 54 


\section{Literaturverzeichnis}

Anandic Medical Systems AG (2007).

http://www.anandic.com/bausteine.net/f/9607/EKGElektrodenPositionierung.p $\mathrm{df} ? \mathrm{fd}=2$

Bertelsen M, Linneberg A, Rosenberg T, Christoffersen N, Vorum H, Gade E, Larsen M (2012): Comorbidity in patients with branch retinal vein occlusion: case-control study. BMJ $\underline{345}$, e7885

Bertelsen M, Linneberg A, Christoffersen N, Vorum H, Gade E, Larsen M (2014): Mortality in patients with central retinal vein occlusion. Ophthalmology $\underline{121}, 637-642$

Brown GC, Shah HG, Magargal LE, Savino PJ (1984): Central retinal vein obstruction and carotid artery disease. Ophthalmology 91 , 1627-1633 Cacciola RR, Guarino F, Polosa R (2007): Relevance of endothelialhaemostatic dysfunction in cigarette smoking. Curr Med Chem $\underline{14}, 1887-$ 1892

Cheung N, Klein R, Wang JJ, Cotch MF, Islam AFM, Klein BEK, Cushman M, Wong TY (2008): Traditional and Novel Cardiovascular Risk Factors for Retinal Vein Occlusion: The Multiethnic Study of Atherosclerosis. Investigative Ophthalmology \& Visual Science $\underline{49}$, 4297-4302

Christiansen CB, Lip GYH, Lamberts M, Gislason G, Torp-Pedersen C, Olesen JB (2013): Retinal vein and artery occlusions: a risk factor for stroke in atrial fibrillation. $\mathrm{J}$ Thromb Haemost $11,1485-1492$

Critchley JA, Capewell S (2003): Mortality risk reduction associated with smoking cessation in patients with coronary heart disease: a systematic review. JAMA 290, 86-97 
Cugati S (2006): Ten-Year Incidence of Retinal Vein Occlusion in an Older Population. Arch Ophthalmol 124, 726

Cugati S, Wang JJ, Knudtson MD, Rochtchina E, Klein R, Klein BEK, Wong TY, Mitchell P (2007): Retinal vein occlusion and vascular mortality: pooled data analysis of 2 population-based cohorts. Ophthalmology 114, 520-524 David R, Zangwill L, Badarna M, Yassur Y (1988): Epidemiology of retinal vein occlusion and its association with glaucoma and increased intraocular pressure. Ophthalmologica $\underline{197}, 69-74$

de Weerd M, Greving JP, de Jong AW, Buskens E, Bots ML (2009):

Prevalence of asymptomatic carotid artery stenosis according to age and sex: systematic review and metaregression analysis. Stroke $\underline{40}, 1105-1113$

Di Capua M, Di Minno MND, Guida A, Loffredo M, Cuccaro C, Coppola A, Izzo R, Palmieri NM, Crispo A, Cerbone AM et al. (2012): Coronary artery disease, cerebral non-fatal ischemic stroke in retinal vein occlusion: an 8-yr follow-up. Nutr Metab Cardiovasc Dis 22, 23-27

Dithmar S, Hansen LL, Holz FG (2003): Venöse retinale Verschlüsse. Der Ophthalmologe $\underline{100}, 561-578$

Elsner D (2006): Therapie der stabilen koronaren Herzkrankheit: Wann Stent, wann medikamentös und wann Operation? Internist (Berl) 47, 1251-1254, 1255-1257

Fegan CD (2002): Central retinal vein occlusion and thrombophilia. Eye (Lond) 16, 98-106

Feist RM, Ticho BH, Shapiro MJ, Farber M (1992): Branch retinal vein occlusion and quadratic variation in arteriovenous crossings. Am J Ophthalmol $\underline{113}$, 664-668 
Feltgen N: Neue invasive Behandlungsmethoden retinaler Venenverschlüsse. Med. Habilschrift Freiburg 2008. Die Verwendung erfolgt mit freundlicher Genehmigung des Autors.

Feltgen N, Franko Zeitz P (2014): Vaskuläre Netzhauterkrankungen als Spiegel generalisierter Gefäßveränderungen. Was können wir voneinander lernen? Ophthalmologe 111, 10-14

Feltgen N, Pielen A, Hansen L, Bertram B, Agostini H, Jaissle GB, Hoerauf H, Stahl A (2010): Intravitreale Medikamenteneingabe bei retinalem Venenverschluss - pathophysiologische Mechanismen und angewandte Substanzen. Klin Monatsbl Augenheilkd 227, 681-693. Die Verwendung erfolgt mit freundlicher Genehmigung des Autors.

Hansen LL: Central Retinal Vein Occlusion. In: Retinal Vascular Disease, hrsg. v. Joussen AM, Gardner TW, Kirchhof B, Ryan SJ. Springer, Berlin/Heidelberg 2007, 443-466

Hansen LL: Retinale Gefäßerkrankungen. In: Retinale Gefäßerkrankungen, hrsg. v. Joussen AM. Springer, Berlin/Heidelberg 2012, 189-215

Harris MI (1998): Diabetes in America: epidemiology and scope of the problem. Diabetes Care 21 Suppl 3, C11-4

Hayreh SS (1980): Hemi-Central Retinal Vein Occlusion. Arch Ophthalmol $\underline{98}, 1600$

Hayreh SS (1994): Retinal vein occlusion. Indian J Ophthalmol 42, 109-132 Hayreh SS (2005): Prevalent misconceptions about acute retinal vascular occlusive disorders. Prog Retin Eye Res 24, 493-519

Heuschmann PU, Di Carlo A, Bejot Y, Rastenyte D, Ryglewicz D, Sarti C, Torrent M, Wolfe, Charles D A (2009): Incidence of stroke in Europe at the beginning of the 21 st century. Stroke $\underline{40}, 1557-1563$ 
Heuschmann PU, Busse O, Wagner M, Endres M, Villringer A, Röther J, Kolominski-Rabas PL, Berger K (2010): Schlaganfallhäufigkeit und Versorgung von Schlaganfallpatienten in Deutschland. Akt Neurol 37, 333340

Hitchings RA, Spaeth GL (1976): Chronic retinal vein occlusion in glaucoma. Br J Ophthalmol 60, 694-699

Ho JD, Liou SW, Lin HC (2009a): Reply. Am J Ophthalmol 147, 1104-1105 Ho JD, Liou SW, Lin HC (2009b): Retinal Vein Occlusion and the Risk of Stroke Development: A Five-year Follow-up Study. Am J Ophthalmol 147, 283-290.e2

Hughes S, Yang H, Chan-Ling T (2000): Vascularization of the human fetal retina: roles of vasculogenesis and angiogenesis. Invest Ophthalmol Vis Sci $\underline{41}, 1217-1228$ Ingerslev J (1999): Thrombophilia, a feature of importance in retinal vein thrombosis? Acta Ophthalmol Scand $\underline{77}, 619-621$ Janssen MCH, den Heijer M, Cruysberg JRM, Wollersheim H, Bredie SJH (2005): Retinal vein occlusion: A form of venous thrombosis or a complication of atherosclerosis? A meta-analysis of thrombophilic factors. Thromb Haemost $\underline{\text { 93, }}$ 1021-1026

Jefferies P, Clemett R, Day T (1993): An anatomical study of retinal arteriovenous crossings and their role in the pathogenesis of retinal branch vein occlusions. Aust N Z J Ophthalmol 21, 213-217

Khan Z, Almeida DRP, Rahim K, Belliveau MJ, Bona M, Gale J (2013): 10Year Framingham risk in patients with retinal vein occlusion: a systematic review and meta-analysis. Can J Ophthalmol (Canadian journal of ophthalmology. Journal canadien d'ophtalmologie) $\underline{48}$, 40-45.e1 
Klatt C, Purtskhvanidze K, Hasselbach H, Treumer F, Hillenkamp J, Roider J (2010): Retrospektive Fallanalyse zum ophthalmologischen und internistischen Risikoprofil von retinalen Gefäßverschlüssen. Ophthalmologe $\underline{107}, 446-451$

Klein R (2008): The 15-Year Cumulative Incidence of Retinal Vein Occlusion. Arch Ophthalmol $\underline{126}, 513$

Klein R, Klein BE, Moss SE, Meuer SM (2000): The epidemiology of retinal vein occlusion: the Beaver Dam Eye Study. Trans Am Ophthalmol Soc $\underline{98}$, 133-41; discussion 141-3

Koizumi H, Ferrara DC, Brue C, Spaide RF (2007): Central retinal vein occlusion case-control study. Am J Ophthalmol 144, 858-863

Kuhli-Hattenbach C, Scharrer I, Lüchtenberg M, Hattenbach LO (2009): Selektives Thrombophiliescreening junger Patienten mit venösen retinalen Gefässverschlüssen. Klin Monbl Augenheilkd 226, 768-773

Kuhli-Hattenbach C, Miesbach W, Scharrer I, Hattenbach L (2011):

Thrombophiliediagnostik und Abklärung systemischer Risikofaktoren bei Patienten mit venösen retinalen Gefäßverschlüssen. Ophthalmologe $\underline{108}$, $104-110$

Lang G (2007): Zentralvenenverschlüsse. Klin Monatsbl Augenheilkd 224, R95

Lang GE, Freissler K (1992): Clinical and fluorescein angiography findings in patients with retinal vein occlusion. A unicenter study of 211 patients. Klin Monbl Augenheilkd 201, 234-239

Lang GE, Spraul CW (1997): Risikofaktoren retinaler Verschlusserkrankungen. Klin Monbl Augenheilkd 211, 217-226 
Lerche R, Wilhelm C, Eifrig B, Richard G (2001): Thrombophiliefaktoren als Auslöser retinaler Gefäßverschlüsse. Der Ophthalmologe $\underline{98}$, 529-534 Marcucci R, Sofi F, Grifoni E, Sodi A, Prisco D (2011): Retinal vein occlusions: a review for the internist. Intern Emerg Med $\underline{6}, 307-314$ Marin-Sanabria EA, Kondoh T, Yamanaka A, Kohmura E (2005): Ultrasonographic screening of carotid artery in patients with vascular retinopathies. Kobe J Med Sci $\underline{51}$, 7-16

Mirshahi A, Feltgen N, Hansen LL, Hattenbach L (2008): Retinal vascular occlusions: an interdisciplinary challenge. Dtsch Arztebl Int 105, 474-479 O'Mahoney PRA (2008): Retinal Vein Occlusion and Traditional Risk Factors for Atherosclerosis. Arch Ophthalmol 126, 692

Patton N, Aslam T, Macgillivray T, Pattie A, Deary IJ, Dhillon B (2005): Retinal vascular image analysis as a potential screening tool for cerebrovascular disease: a rationale based on homology between cerebral and retinal microvasculatures. J Anat (Journal of anatomy) 206, 319-348

Rauh G, Fischereder M, Nasemann J, Muller M, Gruterich M, Spannagl M, Spengel FA (1996): Evaluation of atherosclerosis in patients with central retinal vein occlusion by carotid artery duplex scanning and echocardiography: a prospective case-control study. Eur J Med Res 1, 429432

Rodriguez N, Eliott D (2001): Bilateral central retinal vein occlusion in Eisenmenger syndrome. Am J Ophthalmol 132, 268-269

Rogers S, Mclntosh RL, Cheung N, Lim L, Wang JJ, Mitchell P, Kowalski JW, Nguyen H, Wong TY (2010a): The Prevalence of Retinal Vein Occlusion: Pooled Data from Population Studies from the United States, Europe, Asia, and Australia. Ophthalmology 117, 313-319.e1 
Rogers SL, McIntosh RL, Lim L, Mitchell P, Cheung N, Kowalski JW, Nguyen HP, Wang JJ, Wong TY (2010b): Natural History of Branch Retinal Vein Occlusion: An Evidence-Based Systematic Review. Ophthalmology 117, 1094-1101.e5

Sayag D, Gotzamanis A, Brugniart C, Segal A, Ducasse A, Chambre V, Glacet-Bernard A (2002): Retinal vein occlusion and carotid Doppler imaging. J Fr Ophtalmol 25, 826-830

Seidel H, Stegemann E, Heiss C (2014): Kardiovaskuläre und hämostaseologische Sicht retinaler Gefäßverschlüsse. Ophthalmologe $\underline{111}$, 23-30

Seitz R (1962): Die Netzhautgefässe. Beihefte Klin Monatsbl Augenheilkd 40. Heft, Ferdinand Enke Verlag, Stuttgart

Stahrenberg R, Weber-Krüger M, Seegers J, Edelmann F, Lahno R, Haase B, Mende M, Wohlfahrt J, Kermer P, Vollmann D et al. (2010): Enhanced detection of paroxysmal atrial fibrillation by early and prolonged continuous holter monitoring in patients with cerebral ischemia presenting in sinus rhythm. Stroke $\underline{41}$, 2884-2888

Trenkwalder P (2000): Arterielle Hypertonie - Diagnose und Therapie. Der Internist $\underline{41}, 41-55$

Weinberg D, Dodwell DG, Fern SA (1990): Anatomy of arteriovenous crossings in branch retinal vein occlusion. Am J Ophthalmol 109, 298-302 Werther W, Chu L, Holekamp N, Do DV, Rubio RG (2011): Myocardial Infarction and Cerebrovascular Accident in Patients With Retinal Vein OcclusionRetinal Vein Occlusion. Arch Ophthalmol $\underline{129}, 326$

Wiek J, Schade M, Wiederholt M, Arntz HR, Hansen LL (1990): Haemorheological changes in patients with retinal vein occlusion after isovolaemic haemodilution. $\mathrm{Br} \mathrm{J}$ Ophthalmol $\underline{74}, 665-669$ 
Wong TY, Scott IU (2010): Retinal-Vein Occlusion. N Engl J Med $\underline{363}$, 21352144

Wong TY, Larsen EKM, Klein R, Mitchell P, Couper DJ, Klein BEK, Hubbard LD, Siscovick DS, Sharrett AR (2005): Cardiovascular risk factors for retinal vein occlusion and arteriolar emboli: the Atherosclerosis Risk in Communities \& Cardiovascular Health studies. Ophthalmology $\underline{112}, 540-547$

World Health Organization (2014).

http://apps.who.int/bmi/index.jsp?introPage=intro_3.html Yau JWY, Lee P, Wong TY, Best J, Jenkins A (2008): Retinal vein occlusion: an approach to diagnosis, systemic risk factors and management. Intern Med $\mathrm{J} \underline{38}, 904-910$ 


\section{Präsentationen}

Teile der vorliegenden Dissertation wurden bereits als Poster präsentiert:

02/2013 International Stroke Conference in Honolulu, Hawaii

A. Ammermann, J. Best, I. Arnold, A. Duvinage, R. Stahrenberg, F. Edelmann, J. Wohlfahrt, K. Gröschel, N. Feltgen, R. Wachter The role of paroxysmal atrial fibrillation in patients with retinal artery occlusion (RAO)

09/2013 Kongress der Deutschen Ophthalmologischen Gesellschaft in Berlin
A. Ammermann, J. Best, I. Arnold, A. Duvinage, R. Stahrenberg, F. Edelmann, J. Wohlfahrt, K. Gröschel, N. Feltgen, R. Wachter Risikofaktoren beim retinalen Arterienverschluss: Welche Rolle spielt das Vorhofflimmern? 


\section{Danksagung}

An dieser Stelle möchte ich allen danken ohne die es mir nicht möglich gewesen wäre, diese Arbeit zu vollenden.

Der größte Dank gilt meinem Doktorvater Herrn Professor Dr. med. Nicolas Feltgen für die erstklassige Betreuung, die zuverlässige und engagierte Unterstützung bei allen Fragen zur Erstellung dieser Arbeit.

Herrn Professor Dr. med. Hans Hoerauf, Direktor der Klinik für Augenheilkunde, danke ich für hilfreiche Anregungen und die Möglichkeit, diese Arbeit in seiner Abteilung durchführen zu können.

Besonderer Dank gilt den Patienten und dem gesamten Studienteam, die an der FIND-AF-EYE-Studie teilnahmen. Des Weiteren danke ich Inka Arnold für die gute Zusammenarbeit während der Durchführung der Studie.

Ebenfalls möchte ich mich bei allen Mitarbeitern der kardiologischen Studienambulanz, insbesondere bei Herrn PD Dr. med. Rolf Wachter für die freundliche und fachliche Unterstützung sowie für die Bereitstellung der Studiendaten der FIND-AF-Studie bedanken. 